Iternational Electronic Journal of Algebra

Volume 27 (2020) 237-262

DOI: $10.24330 /$ ieja.663079

\title{
GENERALIZATIONS OF THE ZERO-DIVISOR GRAPH
}

\author{
David F. Anderson and Grace McClurkin \\ Received: 20 May 2019; Accepted: 11 July 2019 \\ Communicated by Sarah Glaz
}

\begin{abstract}
Let $R$ be a commutative ring with $1 \neq 0$ and $Z(R)$ its set of zerodivisors. The zero-divisor graph of $R$ is the (simple) graph $\Gamma(R)$ with vertices $Z(R) \backslash\{0\}$, and distinct vertices $x$ and $y$ are adjacent if and only if $x y=0$. In this paper, we consider generalizations of $\Gamma(R)$ by modifying the vertices or adjacency relations of $\Gamma(R)$. In particular, we study the extended zero-divisor graph $\bar{\Gamma}(R)$, the annihilator graph $A G(R)$, and their analogs for ideal-based and congruence-based graphs.
\end{abstract}

Mathematics Subject Classification (2010): 05C99, 13A15, 13A99

Keywords: Zero-divisor graph, commutative ring with identity

\section{Introduction}

Let $R$ be a commutative ring with $1 \neq 0$, and let $Z(R)$ be its set of zero-divisors. The zero-divisor graph of $R$ is the (simple) graph $\Gamma(R)$ with vertices $Z(R)^{*}=Z(R) \backslash$ $\{0\}$, the set of nonzero zero-divisors of $R$, and distinct vertices $x$ and $y$ are adjacent if and only if $x y=0$. There have been several other "zero-divisor" graphs associated to $R$. The extended zero-divisor graph of $R$ is the (simple) graph $\bar{\Gamma}(R)$ with vertices $Z(R)^{*}$, and distinct vertices $x$ and $y$ are adjacent if and only if $x^{m} y^{n}=0$ for positive integers $m$ and $n$ with $x^{m} \neq 0$ and $y^{n} \neq 0$. The annihilator graph of $R$ is the (simple) graph $A G(R)$ with vertices $Z(R)^{*}$, and distinct vertices $x$ and $y$ are adjacent if and only if $\operatorname{ann}_{R}(x y) \neq \operatorname{ann}_{R}(x) \cup a n n_{R}(y)$ (i.e., $a n n_{R}(x) \cup a n n_{R}(y) \subsetneq a n n_{R}(x y)$ ). These graphs all have the same vertex set $Z(R)^{*}$, and $\Gamma(R) \subseteq \bar{\Gamma}(R) \subseteq A G(R)$.

The ideal-based zero-divisor graph of $R$ with respect to an ideal $I$ of $R$ is the (simple) graph $\Gamma_{I}(R)$ with vertices $Z_{I}(R)=\{x \in R \backslash I \mid x y \in I$ for some $y \in R \backslash I\}$, and distinct vertices $x$ and $y$ are adjacent if and only if $x y \in I$. The ideal-based extended zero-divisor graph of $R$ (with respect to $I$ ) is the (simple) graph $\bar{\Gamma}_{I}(R)$ with vertices $Z_{I}(R)$, and distinct vertices $x$ and $y$ are adjacent if and only if $x^{m} y^{n} \in I$ for positive integers $m$ and $n$ with $x^{m} \notin I$ and $y^{n} \notin I$. The ideal-based annihilator graph of $R$ (with respect to $I$ ) is the (simple) graph $A G_{I}(R)$ with vertices $Z_{I}(R)$, and distinct vertices $x$ and $y$ are adjacent if and only if $(I: x y) \neq(I: x) \cup(I: y)$ 
(i.e., $(I: x) \cup(I: y) \subsetneq(I: x y))$. Thus $\Gamma_{\{0\}}(R)=\Gamma(R), \bar{\Gamma}_{\{0\}}(R)=\bar{\Gamma}(R)$, and $A G_{\{0\}}(R)=A G(R)$. These graphs all have the same vertex set $Z_{I}(R)$, and $\Gamma_{I}(R) \subseteq \bar{\Gamma}_{I}(R) \subseteq A G_{I}(R)$.

More generally, for any (multiplicative) commutative semigroup $S$ with 0 , let $Z(S)=\{x \in S \mid x y=0$ for some $0 \neq y \in S\}$ be the set of zero-divisors of $S$. The zero-divisor graph of $S$ is the (simple) graph $\Gamma(S)$ with vertices $Z(S)^{*}=Z(S) \backslash\{0\}$, and distinct vertices $x$ and $y$ are adjacent if and only if $x y=0$. Thus $\Gamma(R)=\Gamma(S)$, where $S=R$ considered as a monoid under the given ring multiplication (or $S=$ $Z(R)$ ), and $\Gamma_{I}(R)=\Gamma(S)$, where $S=R_{I}$ is the Rees semigroup of $R$ with respect to the ideal $I$. We define the extended zero-divisor graph of $S$ to be the (simple) graph $\bar{\Gamma}(S)$ with vertices $Z(S)^{*}$, and distinct vertices $x$ and $y$ are adjacent if and only if $x^{m} y^{n}=0$ for positive integers $m$ and $n$ with $x^{m} \neq 0$ and $y^{n} \neq 0$; and the annihilator graph of $S$ is the (simple) graph $A G(S)$ with vertices $Z(S)^{*}$, and distinct vertices $x$ and $y$ are adjacent if and only if $a n n_{S}(x y) \neq a n n_{S}(x) \cup a n n_{S}(y)$ (i.e., $\left.\operatorname{ann}_{S}(x) \cup a n n_{S}(y) \subsetneq a n n_{S}(x y)\right)$. These graphs all have the same vertex set $Z(S)^{*}, \Gamma(S) \subseteq \bar{\Gamma}(S)$, and $\bar{\Gamma}(S) \subseteq A G(S)$ if $S \neq Z(S)$ (cf. [3, Theorem 3.1]). In particular, $\Gamma(S) \subseteq \bar{\Gamma}(S) \subseteq A G(S)$ if $S$ has an identity element. Note that $\bar{\Gamma}(R)=\bar{\Gamma}(S)$ and $A G(R)=A G(S)$ for $S=R$ considered as a multipicative monoid, and $\bar{\Gamma}_{I}(R)=\bar{\Gamma}(S)$ and $A G_{I}(R)=A G(S)$ for $S=R_{I}$.

Let $\sim$ be a multiplicative congruence relation on $R$ (i.e., $\sim$ is an equivalence relation on $R$ and $x \sim y$ implies $x z \sim y z$ for $x, y, z \in R)$. Then $R / \sim=\left\{[x]_{\sim} \mid\right.$ $x \in R\}$, the set of $\sim$-congruence classes of $R$, is a commutative monoid under the induced multiplication $[x]_{\sim}[y]_{\sim}=[x y]_{\sim}$ with identity element $[1]_{\sim}$ and zero element $[0]_{\sim}$. (We will often just write $[x]$ for $[x]_{\sim}$ when the context is clear.) Thus $\Gamma_{\sim}(R)=\Gamma(R / \sim)$, called the congruence-based zero-divisor graph of $R$ with respect to $\sim$ (or the $\sim$-zero-divisor graph of $R$ for short), is the (simple) graph with vertices $Z(R / \sim)^{*}=Z(R / \sim) \backslash\left\{[0]_{\sim}\right\}$, and distinct vertices $[x]_{\sim}$ and $[y]_{\sim}$ are adjacent if and only if $[x]_{\sim}[y]_{\sim}=[x y]_{\sim}=[0]_{\sim}$, if and only if $x y \in[0]_{\sim}$. Special cases of $\Gamma_{\sim}(R)$ include the usual zero-divisor graphs $\Gamma(R)$ and $\Gamma(R / I)$, the idealbased zero-divisor graph $\Gamma_{I}(R)$, and the compressed zero-divisor graphs $\Gamma_{E}(R)$ and $\Gamma_{E}(R / I)$ (see Sections 4 and 5 ). This approach clarifies the many isolated results concerning the various zero-divisor graphs spread throughout the literature. We define the extended $\sim$-zero-divisor graph of $R$ to be $\bar{\Gamma}_{\sim}(R)=\bar{\Gamma}(R / \sim)$ and the $\sim$ annihilator graph of $R$ to be $A G_{\sim}(R)=A G(R / \sim)$. These graphs all have the same vertex set $Z(R / \sim)^{*}$, and $\Gamma_{\sim}(R) \subseteq \bar{\Gamma}_{\sim}(R) \subseteq A G_{\sim}(R)$. 
The concept of a zero-divisor graph was introduced by I. Beck ([18]), and then further studied by D. D. Anderson and M. Naseer ([4]). However, they let all the elements of $R$ be vertices of the graph, and they were mainly interested in colorings. Our definition of $\Gamma(R)$ and the emphasis on studying the interplay between the graph-theoretic properties of $\Gamma(R)$ and the ring-theoretic properties of $R$ are from [13]. The extended zero-divisor graph $\bar{\Gamma}(R)$ was given by D. Bennis, J. Mikram, and F. Taraza in [19]. In [17], A. Badawi defined the annihilator graph $A G(R)$. In [31], S. P. Redmond introduced the ideal-based zero-divisor graph $\Gamma_{I}(R)$, and the ideal-based annihilator graph $A G_{I}(R)$ was defined by M. Afkami, N. Hoseini, and K. Khashyarmanesh in [1]. The semigroup zero-divisor graph $\Gamma(S)$ was given by F. R. DeMeyer, T. McKenzie, and K. Schneider in [22], and the semigroup annihilator graph $A G(S)$ was defined by M. Afkhami, K. Khashyarmanesh, and S. M. Sakhdari in [3]. The $\sim$-zero-divisor graph $\Gamma_{\sim}(R)$ was introduced in [12]. For additional information and references about zero-divisor graphs, see [8], [10], [11], [24], [33], and the three survey articles [6], [7], and [21].

Starting with $\Gamma(R)$, we can modify both the vertices and edges to get new "zerodivisor" graphs. In this paper, we study these ideas in more detail. In the second section, we study the ideal-based graphs $\Gamma_{I}(R), \bar{\Gamma}_{I}(R)$, and $A G_{I}(R)$. Results for $\Gamma(R), \bar{\Gamma}(R)$, and $A G(R)$ then follow by letting $I=\{0\}$. In the third section, we study the congruence-based graphs $\Gamma_{\sim}(R), \bar{\Gamma}_{\sim}(R)$, and $A_{\sim}(R)$. We also investigate when $\Gamma_{\sim}(R)$ is complemented or uniquely complemented. In the fourth section, we study compressed graphs. In the final section, we study maps between congruence-based graphs. This extends the work in [12] on $\Gamma_{\sim}(R)$ to $\bar{\Gamma}_{\sim}(R)$ and $A G_{\sim}(R)$.

Throughout, $R$ will be a commutative ring with $1 \neq 0, Z(R)$ its set of zerodivisors and $Z(R)^{*}=Z(R) \backslash\{0\}, \operatorname{nil}(R)$ its set of nilpotent elements, $U(R)$ its group of units, $T(R)=R_{S}$, where $S=R \backslash Z(R)$, its total quotient ring, and $\operatorname{dim}(R)$ its Krull dimension. For $I$ an ideal of $R, \sqrt{I}=\left\{x \in R \mid x^{n} \in I\right.$ for some $n \in \mathbb{N}\}$, and $I$ is a radical ideal if $\sqrt{I}=I$. For $I$ an ideal of $R$ and $x \in R$, let $(I: x)=\{y \in R \mid x y \in I\}$; thus $(0: x)=\operatorname{ann}_{R}(x)$. We say that $R$ is reduced if $\operatorname{nil}(R)=\{0\}$. These concepts extend in the obvious way to semigroups and semigroup ideals. We will often consider $R$ to be a commutative monoid under the ring multiplication. In this case, an (ring) ideal of $R$ is always a semigroup ideal of $R$. However, the converse may fail since the union of (ring) ideals of $R$ is a semigroup ideal of $R$. As usual, we assume that a subring has the same identity element as the ring $R, x^{0}=1$ for $0 \neq x \in R$, and all ring and monoid homomorphisms send 
the identity to the identity. Let $\mathbb{N}, \mathbb{Z}, \mathbb{Z}_{n}, \mathbb{Q}$, and $\mathbb{F}_{q}$ denote the positive integers, integers, integers modulo $n$, rational numbers, and the finite field with $q$ elements, respectively, and $A^{*}=A \backslash\{0\}$. For any undefined ring-theoretic terminology, see [26] or [27]; for semigroups, see [25].

Except for a brief digression in Section 4, we assume that all graphs are simple graphs, i.e., they are undirected graphs with no multiple edges or loops. By abuse of notation, we will often let $G$, rather than $V(G)$, denote the vertices of a graph $G$. Recall that a graph $G$ is connected if there is a path between any two distinct vertices of $G$. For vertices $x$ and $y$ of $G$, let $d(x, y)$ be the length of a shortest path from $x$ to $y(d(x, x)=0$ and $d(x, y)=\infty$ if there is no such path). The diameter of $G$ is $\operatorname{diam}(G)=\sup \{d(x, y) \mid x$ and $y$ are vertices of $G\}$. The girth of $G$, denoted by $\operatorname{gr}(G)$, is the length of a shortest cycle in $G(\operatorname{gr}(G)=\infty$ if $G$ contains no cycles). As usual, $K^{n}$ will denote the complete graph on $n$ vertices, and $K^{m, n}$ will denote the complete bipartite graph on $m, n$ vertices ( $m$ and $n$ may be infinite cardinals). If $m=1$ (or $n=1$ ), then $K^{m, n}$ is called a star graph. A subgraph $H$ of a graph $G$ is an induced subgraph of $G$ if two vertices of $H$ are adjacent in $H$ if and only if they are adjacent in $G$. For graphs $G$ and $G^{\prime}$, a function $f: G \longrightarrow G^{\prime}$ is a graph homomorphism if vertices $x$ and $y$ are adjacent in $G$ implies that $f(x)$ and $f(y)$ are adjacent in $G^{\prime}$. The function $f$ is a graph isomorphism if it is bijective and $f$ and $f^{-1}$ are both graph homomorphisms (i.e., vertices $x$ and $y$ are adjacent in $G$ if and only if $f(x)$ and $f(y)$ are adjacent in $G^{\prime}$ ); in this case, we write $G \cong G^{\prime}$ (again, by abuse of notation, we will often just write $G=G^{\prime}$ when $f$ is a naturally induced graph isomorphism). For a vertex $x$ of a graph $G$, let $N_{G}(x)=\{y \in V(G) \mid y$ is adjacent to $x\}$ and $\bar{N}_{G}(x)=N_{G}(x) \cup\{x\}$. (We will often just write $N(x)$ or $\bar{N}(x)$ when the context is clear.) To avoid trivialities, we will implicitly assume when necessary that our graphs are not the empty graph. A general reference for graph theory is [20].

Many of the results in this paper are from the second-named author's $\mathrm{PhD}$ dissertation ([28]) at The University of Tennessee under the direction of the firstnamed author.

\section{Ideal-based graphs}

In this section, we study the ideal-based graphs $\Gamma_{I}(R), \bar{\Gamma}_{I}(R)$, and $A G_{I}(R)$. These graphs all have common vertex set $Z_{I}(R)$, but different adjacency relations. We will prove results for the general ideal-based case, and then results for $\Gamma(R), \bar{\Gamma}(R)$, and $A G(R)$ follow by letting $I=\{0\}$. Note that $Z_{I}(R)=\emptyset$ if and only 
if $I=R$ or $I$ is a prime ideal of $R$. In particular, $Z(R)^{*}=\emptyset$ if and only if $R$ is an integral domain. Also, if $(I: x y)=(I: x) \cup(I: y)$, then $(I: x) \subseteq(I: y)$ or $(I: y) \subseteq(I: x)$.

There is a strong relationship between $\Gamma_{I}(R)$ and $\Gamma(R / I)$. In fact, $\Gamma_{I}(R)$ may be constructed from $\Gamma(R / I)$ and $I$; see [31, Section 2] for details. A similar relationship and construction exist for $\bar{\Gamma}_{I}(R)$ and $\left.A G_{I}(R)\right)([28])$.

It is clear that $\Gamma_{I}(R)$ is a subgraph of both $\bar{\Gamma}_{I}(R)$ and $A G_{I}(R)$. We first show that $\bar{\Gamma}_{I}(R)$ is also a subgraph of $A G_{I}(R)$.

Theorem 2.1. Let $R$ be a commutative ring with $1 \neq 0$ and $I$ an ideal of $R$. Then $\Gamma_{I}(R) \subseteq \bar{\Gamma}_{I}(R) \subseteq A G_{I}(R)$.

Proof. Clearly $\Gamma_{I}(R) \subseteq \bar{\Gamma}_{I}(R)$. We show that $\bar{\Gamma}_{I}(R) \subseteq A G_{I}(R)$. Let $x$ and $y$ be adjacent vertices in $\bar{\Gamma}_{I}(R)$. Then $x^{m} y^{n} \in I$ for positive integers $m$ and $n$ with $x^{m} \notin I$ and $y^{n} \notin I$. We may assume that $x^{m-1} y^{n} \notin I$ and $x^{m} y^{n-1} \notin I$. Let $z=$ $x^{m-1} y^{n-1}$. Then $z(x y)=x^{m} y^{n} \in I$, but $z x=x^{m} y^{n-1} \notin I$ and $z y=x^{m-1} y^{n} \notin I$; so $z \in(I: x y) \backslash((I: x) \cup(I: y))$. Thus $x$ and $y$ are also adjacent in $A G_{I}(R)$; so $\bar{\Gamma}_{I}(R) \subseteq A G_{I}(R)$.

Corollary 2.2. Let $R$ be a commutative ring with $1 \neq 0$. Then $\Gamma(R) \subseteq \bar{\Gamma}(R) \subseteq$ $A G(R)$.

The following example shows that all possible inclusions may occur.

Example 2.3. Let $R=\mathbb{Z}_{2} \times \mathbb{Z}_{8}$. Note that $(0,2)$ and $(1,2)$ are adjacent in $\bar{\Gamma}(R)$, but not in $\Gamma(R)$; and $(1,2)$ and $(1,4)$ are adjacent in $A G(R)$, but not in $\bar{\Gamma}(R)$. Thus $\Gamma(R) \subsetneq \bar{\Gamma}(R) \subsetneq A G(R)$.

It is easy to check that (1) $\Gamma(R)=\bar{\Gamma}(R)=A G(R)=K^{1}$ for $R=\mathbb{Z}_{4}$, (2) $\Gamma(R) \subsetneq \bar{\Gamma}(R)=A G(R)$ for $R=\mathbb{Z}_{2} \times \mathbb{Z}_{4}$, and $(3) \Gamma(R)=\bar{\Gamma}(R) \subsetneq A G(R)$ for $R=\mathbb{Z}_{2} \times \mathbb{Z}_{2} \times \mathbb{Z}_{2}$.

Remark 2.4. One is tempted to define the extended annihilator graph $\overline{A G}(R)$ with vertices $Z(R)^{*}$, and distinct vertices $x$ and $y$ are adjacent if and only if $\operatorname{ann}_{R}\left(x^{m} y^{n}\right) \neq \operatorname{ann}_{R}\left(x^{m}\right) \cup a n n_{R}\left(y^{n}\right)$ for positive integers $m$ and $n$. However, $\overline{A G}(R)=A G(R)$. Clearly $A G(R) \subseteq \overline{A G}(R)$. For the reverse inclusion, let $x$ and $y$ be adjacent vertices in $\overline{A G}(R)$. Then $\operatorname{ann}_{R}\left(x^{m} y^{n}\right) \neq \operatorname{ann}_{R}\left(x^{m}\right) \cup a n n_{R}\left(y^{n}\right)$ for positive integers $m$ and $n$, and thus $z x^{m} y^{n}=0$, but $z x^{m} \neq 0$ and $z y^{n} \neq 0$, for some $z \in R$. We may assume that $z x^{m} y^{n-1} \neq 0$ and $z x^{m-1} y^{n} \neq 0$. Thus $z x^{m-1} y^{n-1} \in \operatorname{ann}_{R}(x y) \backslash\left(a n n_{R}(x) \cup a n n_{R}(y)\right)$, and hence $x$ and $y$ are also adjacent in $A G(R)$. Thus $\overline{A G}(R)=A G(R)$. In a similar manner, we can define $\overline{A G}_{I}(R)$, and as above, $\overline{A G}_{I}(R)=A G_{I}(R)$. 
Although distinct, nonzero, nilpotent elements $x$ and $y$ need not be adjacent in $\Gamma(R)$, we have $d(x, y) \leq 2$ in $\Gamma(R)$, and they are always adjacent in $A G(R)$. We generalize this to $\Gamma_{I}(R)$ and $A G_{I}(R)$.

Theorem 2.5. Let $R$ be a commutative ring with $1 \neq 0, I$ an ideal of $R$, and $x, y \in \sqrt{I} \backslash I$ with $x \neq y$.

(a) $d(x, y) \leq 2$ in $\Gamma_{I}(R)$.

(b) $x$ and $y$ are adjacent in $A G_{I}(R)$.

Proof. (a) Let $x, y \in \sqrt{I} \backslash I$ be distinct with $x y \notin I$. Let $m$ and $n$ be the least positive integers such that $x^{m}, y^{n} \in I$. Choose $j$ to be the greatest nonnegative integer such that $z=x^{m-1} y^{j} \notin I$. Then $x z=x^{m} y^{j} \in I$ and $z y=x^{m-1} y^{j+1} \in I$ by choice of $j$; so $d(x, y)=2$ in $\Gamma_{I}(R)$.

(b) Let $x, y \in \sqrt{I} \backslash I$ be distinct. Let $m$ and $n$ be the least positive integers such that $x^{m}, y^{n} \in I$; so $x^{m} y^{n} \in I$. If $x^{i} y^{j} \in I$ for some $1 \leq i \leq m-1,1 \leq j \leq n-1$, then $x$ and $y$ are adjacent in $\bar{\Gamma}_{I}(R)$, and hence in $A G_{I}(R)$ by Theorem 2.1. So we may assume that $x^{i} y^{j} \notin I$ for every $1 \leq i \leq m-1$ and $1 \leq j \leq n-1$. In particular, $x^{m-1} y^{n-1}, x^{m-1} y^{n-2}, x^{m-2} y^{n-1} \notin I$. Let $z=x^{m-1} y^{n-2}+x^{m-2} y^{n-1}$. Then $z x y=x^{m} y^{n-1}+x^{m-1} y^{n} \in I$, and $z x=x^{m} y^{n-2}+x^{m-1} y^{n-1} \notin I$ since $x^{m} y^{n-2} \in I$ and $x^{m-1} y^{n-1} \notin I$. Similarly, $z y=x^{m-1} y^{n-1}+x^{m-2} y^{n} \notin I$. Thus $(I: x y) \neq(I: x) \cup(I: y)$; so $x$ and $y$ are adjacent in $A G_{I}(R)$.

Corollary 2.6. Let $R$ be a commutative ring with $1 \neq 0$ and $0 \neq x, y \in \operatorname{nil}(R)$ with $x \neq y$.

(a) ([5, Lemma 3.11]) $d(x, y) \leq 2$ in $\Gamma(R)$.

(b) $([17$, Theorem 3.10]) $x$ and $y$ are adjacent in $A G(R)$.

Example 2.7. Let $R=\mathbb{Z}_{2}[X, Y] /\left(X^{2}, Y^{2}\right)=\mathbb{Z}_{2}[x, y]$. Then $0 \neq x, y \in n i l(R)$ are distinct and adjacent in $A G(R)$, but are not adjacent in $\Gamma(R)$ or $\bar{\Gamma}(R)$.

The following theorem gives conditions for equality in Theorem 2.1. (The proof of part (a) (resp., part (b)) patches some gaps in the proof of [19, Theorem 2.1] (resp., [30, Theorem 3.1]).) As in [16], an ideal $I$ of $R$ is a 2-absorbing ideal of $R$ if whenever $x y z \in I$ for $x, y, z \in R$, then $x y \in I, x z \in I$, or $y z \in I$.

Theorem 2.8. Let $R$ be a commutative ring with $1 \neq 0$ and $I$ an ideal of $R$.

(a) $\bar{\Gamma}_{I}(R)=\Gamma_{I}(R)$ if and only if

(1) $x \in \sqrt{I}$ implies $x^{2} \in I$, and

(2) $x \in R \backslash \sqrt{I}$ implies $\left(I: x^{2}\right)=(I: x)$. 
In particular, $\bar{\Gamma}_{I}(R)=\Gamma_{I}(R)$ when $I$ is a radical ideal of $R$.

(b) $A G_{I}(R)=\Gamma_{I}(R)$ if and only if $I$ is a 2-absorbing ideal of $R$.

Proof. (a) Suppose that $\bar{\Gamma}_{I}(R)=\Gamma_{I}(R)$. First, let $x \in \sqrt{I}$. If $x^{n} \in I$, but $x^{n-1} \notin I$ for $n \geq 3$, let $y=x\left(1+x^{n-2}\right)$. Then $y \in \sqrt{I} \backslash I(y \notin I$ since $y \in I$ implies $\left.x^{2}=x y-x^{n} \in I\right), x \neq y$, and $x$ and $y$ are adjacent in $\bar{\Gamma}_{I}(R)$ since $x^{n-1} y=x^{n}\left(1+x^{n-2}\right) \in I$. Thus $x$ and $y$ are also adjacent in $\Gamma_{I}(R)$; so $x y \in I$. Hence $x^{2}=x y-x^{n} \in I$, a contradiction; so $x^{2} \in I$ and (1) holds. Next, let $x \in R \backslash \sqrt{I}$ and $y \in\left(I: x^{2}\right)$. If $y \in I$, then $y \in(I: x)$. If $x=y$, then $x^{3} \in I$, a contradiction. So assume that $y \notin I$ and $x \neq y$. Then $x^{2} y \in I$; so $x$ and $y$ are adjacent in $\bar{\Gamma}_{I}(R)$. Hence they are also adjacent in $\Gamma_{I}(R)$; so $x y \in I$. Thus $\left(I: x^{2}\right) \subseteq(I: x)$; so (2) holds.

Conversely, suppose that (1) and (2) hold. We have $\Gamma_{I}(R) \subseteq \bar{\Gamma}_{I}(R)$ by Theorem 2.1. For the reverse inclusion, let $x$ and $y$ be adjacent vertices in $\bar{\Gamma}_{I}(R)$. Then $x^{m} y^{n} \in I$ for positive integers $m$ and $n$ with $x^{m} \notin I$ and $y^{n} \notin I$. If $x \in \sqrt{I}$, then $m=1$ by (1). Thus $x y^{n} \in I$. If in addition, $y \in \sqrt{I}$, then $n=1$ by (1) again; so $x y \in I$. If $y \notin \sqrt{I}$, then $x \in\left(I: y^{n}\right)=(I: y)$ by (2) and induction. Thus $x y \in I$. Similarly, $x y \in I$ if $x \notin \sqrt{I}$. So in every case, $x y \in I$, and hence $x$ and $y$ are also adjacent in $\Gamma_{I}(R)$. Thus $\bar{\Gamma}_{I}(R)=\Gamma_{I}(R)$.

The "in particular" statement is clear.

(b) First, suppose that $A G_{I}(R)=\Gamma_{I}(R)$. Let $x y z \in I$ for $x, y, z \in R$. There are three cases.

(1) Suppose that $x=y=z$; then $x^{3} \in I$. If $x^{2} \notin I$, then $x$ and $w=x(1+x)$ are distinct adjacent vertices in $A G_{I}(R)$, and hence are also adjacent in $\Gamma_{I}(R)$. Thus $x w \in I$; so $x^{2}=x w-x^{3} \in I$, a contradiction. Hence $x y=x^{2} \in I$.

(2) Suppose that $x=y$ and $z$ are distinct; then $x^{2} z \in I$. Suppose that $x^{2} \notin I$. If $x z \notin I$, then $x \in(I: x z) \backslash((I: x) \cup(I: z))$. Thus $x$ and $z$ are adjacent vertices in $A G_{I}(R)$, and hence are also adjacent in $\Gamma_{I}(R)$. Thus $x z \in I$, a contradiction. So $x z \in I$.

(3) Suppose that $x, y, z$ are all distinct, and $x z \notin I$ and $y z \notin I$. Then $z \in(I$ : $x y) \backslash((I: x) \cup(I: y))$; so $x$ and $y$ are adjacent in $A G_{I}(R)$. Thus $x$ and $y$ are also adjacent in $\Gamma_{I}(R)$; so $x y \in I$.

Thus $I$ is a 2 -absorbing ideal of $R$.

Conversely, suppose that $I$ is a 2-absorbing ideal of $R$. We have $\Gamma_{I}(R) \subseteq A G_{I}(R)$ by Theorem 2.1. For the reverse inclusion, let $x$ and $y$ be adjacent vertices in $A G_{I}(R)$. Then there is a $z \in(I: x y) \backslash((I: x) \cup(I: y))$. Thus $x y z \in I, x z \notin I$, 
and $y z \notin I$. Hence $x y \in I$ since $I$ is a 2-absorbing ideal of $R$, and thus $x$ and $y$ are also adjacent in $\Gamma_{I}(R)$. Hence $A G_{I}(R)=\Gamma_{I}(R)$.

Corollary 2.9. Let $R$ be a commutative ring with $1 \neq 0$.

(a) ([19, Theorem 2.1]) $\bar{\Gamma}(R)=\Gamma(R)$ if and only if

(1) $x \in \operatorname{nil}(R)$ implies $x^{2}=0$, and

(2) $x \in R \backslash \operatorname{nil}(R)$ implies ann $\operatorname{an}_{R}\left(x^{2}\right)=\operatorname{ann}_{R}(x)$.

In particular, $\bar{\Gamma}(R)=\Gamma(R)$ when $R$ is reduced.

(b) ([30, Theorem 3.1]) $A G(R)=\Gamma(R)$ if and only if $\{0\}$ is a 2-absorbing ideal of $R$.

In [11, Theorem 2.2] (resp., [2, Theorem 4.10]), it was shown that $\Gamma(T(R)) \cong$ $\Gamma(R)$ (resp., $A G(T(R)) \cong A G(R))$. We next show that $\bar{\Gamma}(T(R)) \cong \bar{\Gamma}(R)$ as well.

Theorem 2.10. Let $R$ be a commutative ring with $1 \neq 0$ and total quotient ring $T(R)$. Then $\Gamma(T(R)) \cong \Gamma(R), \bar{\Gamma}(T(R)) \cong \bar{\Gamma}(R)$, and $A G(T(R)) \cong A G(R)$.

Proof. Let $T=T(R)$. We need only show that $\bar{\Gamma}(T) \cong \bar{\Gamma}(R)$. Let $\varphi: Z(R)^{*} \longrightarrow$ $Z(T)^{*}$ be the bijection defined in the proof of [11, Theorem 2.2], and let $\sim_{R}$ (resp., $\sim_{T}$ ) be the congruence relation defined on $Z(R)^{*}$ (resp., $\left.Z(T)^{*}\right)$ by $a \sim_{R} b$ (resp., $\left(a \sim_{T} b\right) \Leftrightarrow a n n_{R}(a)=a n n_{R}(b)\left(\right.$ resp., $\left.a n n_{T}(a)=a n n_{T}(b)\right)$. We need to show that vertices $x$ and $y$ are adjacent in $\bar{\Gamma}(R)$ if and only if $\varphi(x)$ and $\varphi(y)$ are adjacent in $\bar{\Gamma}(T)$, i.e., $x^{m} y^{n}=0$ for positive integers $m, n$ with $x^{m} \neq 0$ and $y^{n} \neq 0$ if and only if $\varphi(x)^{m} \varphi(y)^{n}=0$ for positive integers $m, n$ with $\varphi(x)^{m} \neq 0$ and $\varphi(y)^{n} \neq 0$. The proof of [11, Theorem 2.2] shows that $\varphi\left(x^{m}\right) \varphi\left(y^{n}\right)=0$ when $x^{m} y^{n}=0$. Since $\sim_{R}$ and $\sim_{T}$ are congruence relations, we have $\varphi\left(x^{m}\right) \sim_{T} \varphi(x)^{m}$ and $\varphi\left(y^{n}\right) \sim_{T} \varphi(y)^{n}$. Thus $\varphi(x)^{m} \varphi(y)^{n}=0$. Clearly, $x^{m} \neq 0 \Leftrightarrow \varphi\left(x^{m}\right) \neq 0 \Leftrightarrow \varphi(x)^{m} \neq 0$. So the result holds.

Probably the two best known properties of $\Gamma(R)$ are that $\Gamma(R)$ is connected with $\operatorname{diam}(\Gamma(R)) \in\{0,1,2,3\}$ ([13, Theorem 2.3]) and $\operatorname{gr}(\Gamma(R)) \in\{3,4, \infty\}([13$, Theorem 2.4], [23, Theorem 1.6], [29, (1.4)]). Also, $\operatorname{diam}(\bar{\Gamma}(R)) \in\{0,1,2,3\}$ ([19, Theorem 3.1]), $\operatorname{gr}(\bar{\Gamma}(R)) \in\{3,4, \infty\}([19$, Theorem 4.1]), $\operatorname{diam}(A G(R)) \in\{0,1,2\}$ ([17, Theorem 2.2]), and $\operatorname{gr}(A G(R)) \in\{3,4, \infty\}$ ([17, Corollary 2.11]). These results also hold for the ideal-based graphs and have been investigated in [31] and [15] for $\Gamma_{I}(R)$ and in [1] for $A G_{I}(R)$.

Theorem 2.11. Let $R$ be a commutative ring with $1 \neq 0$ and $I$ an ideal $R$.

(a) $\Gamma_{I}(R), \bar{\Gamma}_{I}(R)$, and $A G_{I}(R)$ are connected.

(b) $0 \leq \operatorname{diam}\left(A G_{I}(R)\right) \leq \operatorname{diam}\left(\bar{\Gamma}_{I}(R)\right) \leq \operatorname{diam}\left(\Gamma_{I}(R)\right) \leq 3$. 
(c) $\operatorname{diam}\left(A G_{I}(R)\right) \in\{0,1,2\}$.

(d) $\Gamma_{I}(R), \bar{\Gamma}_{I}(R)$, and $A G_{I}(R)$ have girth 3,4 , or $\infty$. Moreover, $\operatorname{gr}\left(A G_{I}(R)\right) \leq$ $\operatorname{gr}\left(\bar{\Gamma}_{I}(R)\right) \leq \operatorname{gr}\left(\Gamma_{I}(R)\right)$.

Proof. (a) and (b) are clear since $\Gamma_{I}(R)$ is connected with $\operatorname{diam}\left(\Gamma_{I}(R)\right) \in\{0,1,2,3\}$ ([31, Theorem 2.4]), all three graphs have the same vertex set $Z_{I}(R)$, and $\Gamma_{I}(R) \subseteq$ $\bar{\Gamma}_{I}(R) \subseteq A G_{I}(R)$ by Theorem 2.1 .

(c) By part (b), we need only show that if $d(x, y)=3$ in $\Gamma_{I}(R)$, then $x$ and $y$ are adjacent in $A G_{I}(R)$. Since $d(x, y)=3$ in $\Gamma_{I}(R)$, there are $a, b \in R \backslash I$ such that $x a, a b, b y \in I$ and $x b, a y \notin I$. Thus $(I: x) \nsubseteq(I: y)$ and $(I: y) \nsubseteq(I: x)$; so $(I: x y) \neq(I: x) \cup(I: y)$. Hence $x$ and $y$ are adjacent in $A G_{I}(R)$. (Also, see the proof of Theorem 3.3(c)).

(d) We have $\operatorname{gr}\left(\Gamma_{I}(R)\right) \in\{3,4, \infty\}\left(\left[31\right.\right.$, Lemma 5.1]) and $\operatorname{gr}\left(A G_{I}(R)\right) \in\{3,4, \infty\}$ ([1, Theorem 1.7]). If $I=\{0\}$, then $\bar{\Gamma}_{I}(R)=\bar{\Gamma}(R)$ has girth 3,4 , or $\infty$ ([19, Theorem 4.1]). So we may assume that $I$ is a nonzero, proper ideal of $R$ that is not prime. If $g r\left(\Gamma_{I}(R)\right) \in\{3,4\}$, then clearly $g r\left(\bar{\Gamma}_{I}(R)\right) \in\{3,4\}$ since $\Gamma_{I}(R) \subseteq \bar{\Gamma}_{I}(R)$. If $\operatorname{gr}\left(\Gamma_{I}(R)\right)=\infty$, then $\Gamma_{I}(R)=K^{2}$ by (the proof of) [15, Theorem 3.1]. Thus $\bar{\Gamma}_{I}(R)=K^{2} ;$ so $\operatorname{gr}\left(\bar{\Gamma}_{I}(R)\right)=\infty$. Hence $\operatorname{gr}\left(\bar{\Gamma}_{I}(R)\right) \in\{3,4, \infty\}$.

The "moreover" statement is clear since $\Gamma_{I}(R) \subseteq \bar{\Gamma}_{I}(R) \subseteq A G_{I}(R)$.

Corollary 2.12. Let $R$ be a commutative ring with $1 \neq 0$.

(a) $\Gamma(R), \bar{\Gamma}(R)$, and $A G(R)$ are connected.

(b) $0 \leq \operatorname{diam}(A G(R)) \leq \operatorname{diam}(\bar{\Gamma}(R)) \leq \operatorname{diam}(\Gamma(R)) \leq 3$.

(c) $\operatorname{diam}(A G(R)) \in\{0,1,2\}$.

(d) $\Gamma(R), \bar{\Gamma}(R)$, and $A G(R)$ have girth 3,4 , or $\infty$. Moreover, $\operatorname{gr}(A G(R)) \leq$ $\operatorname{gr}(\bar{\Gamma}(R)) \leq \operatorname{gr}(\Gamma(R))$.

The following relationship between $\operatorname{diam}(\Gamma(R))$ and $\operatorname{diam}(A G(R))$ will be needed in Theorem 2.14.

Theorem 2.13. Let $R$ be a commutative ring with $1 \neq 0$.

(a) If $A G(R)$ is complete and $R \neq \mathbb{Z}_{2} \times \mathbb{Z}_{2}$, then $Z(R)$ is an ideal of $R$.

(b) If $A G(R)$ is complete, then $\operatorname{diam}(\Gamma(R)) \leq 2$. In particular, $0 \leq \operatorname{diam}(\Gamma(R)) \leq$ $\operatorname{diam}(A G(R))+1$.

Proof. (a) This follows from the proof of [2, Proposition 4.1].

(b) Suppose that $A G(R)$ is complete. We may assume that $\left|Z(R)^{*}\right| \geq 3$; so $R \neq \mathbb{Z}_{2} \times \mathbb{Z}_{2}$. Thus $Z(R)$ is an ideal of $R$ by part (a). Let $x, y \in Z(R)^{*}$ be distinct. Then $x-y \in Z(R)$; so there is a $0 \neq z \in R$ with $z(x-y)=0$. We may assume 
that $z \neq x$. Since $A G(R)$ is complete, $z$ and $x$ are adjacent in $A G(R)$. Hence $\operatorname{ann}_{R}(z x) \neq \operatorname{ann}_{R}(z) \cup \operatorname{ann}_{R}(x)$; so there is a $0 \neq t \in R$ with $t(z x)=0, t z \neq 0$, and $t x \neq 0$. Thus $w=t z \in Z(R)^{*}$ and $w x=w y=0$; so $d(x, y) \leq 2$ in $\Gamma(R)$. Hence $\operatorname{diam}(\Gamma(R)) \leq 2$.

The "in particular" statement is clear.

We next give a more careful comparison of the diameter and girth for the three zero-divisor graphs.

Let $a=\operatorname{diam}(\Gamma(R)), b=\operatorname{diam}(\bar{\Gamma}(R)), c=\operatorname{diam}(A G(R))$. Then $0 \leq c \leq b \leq a \leq$ 3 and $0 \leq c \leq 2$ by Corollary 2.12, and $0 \leq a \leq c+1$ by Theorem 2.13. There are 9 integer-tuples $(a, b, c)$ that satisfy these three conditions. We show that 7 of them can be realized as $(\operatorname{diam}(\Gamma(R)), \operatorname{diam}(\bar{\Gamma}(R)), \operatorname{diam}(A G(R)))$ for some commutative ring $R$ with $1 \neq 0$.

Theorem 2.14. Let $a, b, c \in \mathbb{N} \cup\{0\}$. Then there is a commutative ring $R$ with $1 \neq 0$ such that $(a, b, c)=(\operatorname{diam}(\Gamma(R)), \operatorname{diam}(\bar{\Gamma}(R)), \operatorname{diam}(A G(R)))$ if and only if $(a, b, c)$ is $(0,0,0),(1,1,1),(2,1,1),(2,2,1),(2,2,2),(3,2,2)$, or $(3,3,2)$.

Proof. If $a=\operatorname{diam}(\Gamma(R))=0$, then $\Gamma(R)=\bar{\Gamma}(R)=A G(R)=K^{1}$ and $R \cong \mathbb{Z}_{4}$ or $\mathbb{Z}_{2}[X] /\left(X^{2}\right)$. Thus, $b=\operatorname{diam}(\bar{\Gamma}(R))=0$ and $c=\operatorname{diam}(A G(R))=0$; so $(a, b, c)=$ $(0,0,0)$ can be realized.

If $a=\operatorname{diam}(\Gamma(R))=1$, then $\Gamma(R)=\bar{\Gamma}(R)=A G(R)=K^{n}$ for some $n \geq 2$. Thus $b=\operatorname{diam}(\bar{\Gamma}(R))=1$ and $c=\operatorname{diam}(A G(R))=1$. Hence, for example, $(a, b, c)=(1,1,1)$ can be realized using $R=\mathbb{Z}_{2} \times \mathbb{Z}_{2}$. Moreover, $(1,1,0)$ and $(1,0,0)$ cannot be realized.

Let $a=\operatorname{diam}(\Gamma(R))=2$. Then $1 \leq c \leq b \leq 2$. Let $R=\mathbb{Z}_{8}$. Then $\Gamma(R)=K^{1,2}$ has diameter 2 , and $\bar{\Gamma}(R)=A G(R)=K^{3}$ each have diameter 1 . Thus $(a, b, c)=$ $(2,1,1)$ can be realized. Next, let $S=\mathbb{Z}_{2}[X, Y] /\left(X^{2}, Y^{2}\right)$. Then $\Gamma(S)=\bar{\Gamma}(S)$ each have diameter 2 and $A G(S)=K^{7}$ has diameter 1 . Hence $(a, b, c)=(2,2,1)$ can be realized. Finally, let $T=\mathbb{Z}_{6}$. Then $\Gamma(T)=\bar{\Gamma}(T)=A G(T)=K^{1,2}$; so each graph has diameter 2. Thus $(a, b, c)=(2,2,2)$ can be realized. By Theorem 2.13(b), the $(2,2,0),(2,1,0)$, and $(2,0,0)$ cases cannot be realized.

Let $a=\operatorname{diam}(\Gamma(R))=3$. Let $R=\mathbb{Z}_{2} \times \mathbb{Z}_{4}$. Then $\operatorname{diam}(\Gamma(R))=3$ and $\bar{\Gamma}(R)=$ $A G(R)$ each have diameter 2 . Thus $(a, b, c)=(3,2,2)$ can be realized. Next, let $S=\mathbb{Z}_{2} \times \mathbb{Z}_{2} \times \mathbb{Z}_{2}$. Then $\Gamma(S)=\bar{\Gamma}(S)$ each have diameter 3 and $\operatorname{diam}(A G(S))=2$. Thus $(a, b, c)=(3,3,2)$ can be realized. By Theorem 2.13(b), the $(3,1,1),(3,2,1)$, and $(3,3,1)$ cases cannot be realized. 
Now, let $a=\operatorname{gr}(\Gamma(R)), b=\operatorname{gr}(\bar{\Gamma}(R)), c=\operatorname{gr}(A G(R))$. Then $a, b, c \in\{3,4, \infty\}$ and $3 \leq c \leq b \leq a \leq \infty$ by Corollary 2.12. There are 10 tuples $(a, b, c)$ with $a, b, c \in \mathbb{N} \cup\{\infty\}$ that satisfy these two conditions. We show that 6 of them can be realized as $(\operatorname{gr}(\Gamma(R)), \operatorname{gr}(\bar{\Gamma}(R)), \operatorname{gr}(A G(R)))$ for some commutative ring $R$ with $1 \neq 0$. As an added bonus, the proof shows which rings have these given girth tuples (cf. [19, Theorems 4.5 and 4.6]).

Theorem 2.15. Let $a, b, c \in \mathbb{N} \cup\{\infty\}$. Then there is a commutative ring $R$ with $1 \neq 0$ such that $(a, b, c)=(\operatorname{gr}(\Gamma(R)), \operatorname{gr}(\bar{\Gamma}(R)), \operatorname{gr}(A G(R)))$ if and only if $(a, b, c)$ is $(3,3,3),(4,4,3),(4,4,4),(\infty, 3,3),(\infty, 4,4)$, or $(\infty, \infty, \infty)$.

Proof. We have $\operatorname{gr}(\Gamma(R)) \in\{3,4, \infty\}$. We handle each case separately.

(1) $\operatorname{gr}(\Gamma(R))=3$.

In this case, $\operatorname{gr}(\Gamma(R))=\operatorname{gr}(\bar{\Gamma}(R))=\operatorname{gr}(A G(R))=3$. For example, let $R=$ $\mathbb{F}_{4}[X] /\left(X^{2}\right)$. Then $\Gamma(R)=\bar{\Gamma}(R)=A G(R)=K^{3}$; so all three graphs have girth 3 . Thus $(a, b, c)=(3,3,3)$ can be realized.

(2) $\operatorname{gr}(\Gamma(R))=4$.

If $R$ is reduced, then $T(R)=K_{1} \times K_{2}$ for fields $K_{1}$ and $K_{2}$ with $\left|K_{i}\right| \geq 3$ ([14, Theorem 2.2]). Thus, $\Gamma(R)=\bar{\Gamma}(R)=A G(R)=K^{m, n}$, where $m=\left|K_{1}\right|-1 \geq 2$ and $n=\left|K_{2}\right|-1 \geq 2$. So each graph has girth 4 . For example, let $R=\mathbb{Z}_{3} \times \mathbb{Z}_{3}$. Then $\Gamma(R)=\bar{\Gamma}(R)=A G(R)=K^{2,2}$; so all three graphs have girth 4 . Thus $(a, b, c)=(4,4,4)$ can be realized.

If $R$ is not reduced, then $R \cong D \times B$, where $D$ is an integral domain with $|D| \geq 3$ and $B$ is $\mathbb{Z}_{4}$ or $\mathbb{Z}_{2}[X] /\left(X^{2}\right)$ ([14, Theorem 2.3]). Thus $\bar{\Gamma}(R)=\Gamma(R)$; so each graph has girth 4 . However, $\operatorname{gr}(A G(R))=3$ since $(0, f)-(d, f)-(e, f)-(0, f)$ is a 3 -cycle in $A G(R)$ for distinct $0 \neq d, e \in D$ and $0 \neq f \in B$ with $f^{2}=0$. For example, let $R=\mathbb{Z}_{3} \times \mathbb{Z}_{4}$. Hence $(a, b, c)=(4,4,3)$ can be realized, and $(4,3,3)$ cannot be realized.

(3) $\operatorname{gr}(\Gamma(R))=\infty$.

If $R$ is reduced, then $T(R)=\mathbb{Z}_{2} \times K$ for $K$ a field ([14, Theorem 2.4]). Thus, $\Gamma(R)=\bar{\Gamma}(R)=A G(R)=K^{1, n}$, where $n=|K|-1$. In this case, $\operatorname{gr}(\Gamma(R))=$ $\operatorname{gr}(\bar{\Gamma}(R))=\operatorname{gr}(A G(R))=\infty$. For example, let $R=\mathbb{Z}_{2} \times \mathbb{Z}_{2}$. Then $\Gamma(R)=\bar{\Gamma}(R)=$ $A G(R)=K^{1,1}=K^{2}$; so all three graphs have girth $\infty$. Hence $(a, b, c)=(\infty, \infty, \infty)$ can be realized.

If $R$ is not reduced, there are three cases $\left(\left[14\right.\right.$, Theorem2.5]). Let $B=\mathbb{Z}_{4}$ or $\mathbb{Z}_{2}[X] /\left(X^{2}\right)$.

(i) $R \cong B$. In this case, $\Gamma(R)=\bar{\Gamma}(R)=A G(R)=K^{1}$. So all three graphs have infinite girth. Thus, again, $(a, b, c)=(\infty, \infty, \infty)$ can be realized. 
(ii) $R \cong \mathbb{Z}_{2} \times B$. In this case, $\Gamma(R) \subsetneq \bar{\Gamma}(R)=A G(R)$, and $A G(R)$ has the 4-cycle $(1,0)-(0,1)-(1, d)-(0, d)-(1,0)$, where $0 \neq d \in B$ with $d^{2}=0$, and no 3 -cycles. Thus $(a, b, c)=(\infty, 4,4)$ can be realized.

(iii) $\Gamma(R)$ is a star graph with $|R|=8,|R|=9$, or $\Gamma(R)$ is infinite with center $x$ and $\operatorname{nil}(R)=\{0, x\}$ with $x^{2}=0$.

For $|R|=8$ (e.g., $R=\mathbb{Z}_{8}$ ), we have $\Gamma(R)=K^{1,2}$ and $\bar{\Gamma}(R)=A G(R)=K^{3}$ (cf. [11, Remark 3.12]). Thus $\operatorname{gr}(\Gamma(R))=\infty$ and $\operatorname{gr}(\bar{\Gamma}(R))=\operatorname{gr}(A G(R))=3$. Thus $(a, b, c)=(\infty, 3,3)$ can be realized. For $|R|=9$ (e.g., $\left.R=\mathbb{Z}_{9}\right)$, we have $\Gamma(R)=\bar{\Gamma}(R)=A G(R)=K^{1,1}=K^{2}$ (cf. [11, Remark 3.12]). Hence, again, $(a, b, c)=(\infty, \infty, \infty)$ can be realized.

For $\Gamma(R)$ an infinite star graph, we have $\Gamma(R)=\bar{\Gamma}(R)=A G(R)=K^{1, m}$ for some infinite cardinal $m$. (Let $d, e \in R \backslash\{0, x\}$ be distinct vertices. Then $\operatorname{ann}_{R}(d)=$ $\operatorname{ann}_{R}(e)=\{0, x\}$ since $d, e \notin \operatorname{nil}(R)$ and $0 \neq d e \neq x$; so $\operatorname{ann}_{R}(d e)=\{0, x\}$. Hence $A G(R)=\Gamma(R)$.) So all three graphs have infinite girth. Thus, again, $(a, b, c)=(\infty, \infty, \infty)$ can be realized.

Moreover, $(a, b, c)=(\infty, \infty, 4),(\infty, \infty, 3)$, and $(\infty, 4,3)$ cannot be realized.

\section{Congruence-based graphs}

In this section, we consider congruence-based graphs. For a more detailed account of the $\sim$-zero-divisor graph $\Gamma_{\sim}(R)$, see [12]. In Section 5, we consider maps between congruence-based graphs.

We first define the congruence-based analogs of $\bar{\Gamma}(R)$ and $A G(R)$. Let $\sim$ be a multiplicative congruence relation on $R$. Then $R / \sim$ is a commutative monoid with zero $[0]_{\sim}$, and $I=[0]_{\sim}$ is a semigroup ideal of $R$. We define the extended $\sim$ zero-divisor graph of $R$ to be $\bar{\Gamma}_{\sim}(R)=\bar{\Gamma}(R / \sim)$, and the annihilator $\sim$-zero-divisor graph of $R$ to be $A G_{\sim}(R)=A G(R / \sim)$. These graphs all have the same vertex set $Z(R / \sim)^{*}=Z(R / \sim) \backslash\left\{[0]_{\sim}\right\}$, and distinct vertices $[x]_{\sim}$ and $[y]_{\sim}$ are adjacent in $\Gamma_{\sim}(R)\left(\operatorname{resp} ., \bar{\Gamma}_{\sim}(R), A G_{\sim}(R)\right)$ if and only if $[x]_{\sim}[y]_{\sim}=[0]_{\sim}\left(\operatorname{resp} .,[x]_{\sim}^{m}[y]_{\sim}^{n}=[0]_{\sim}\right.$ for positive integers $m$ and $n$ with $[x]_{\sim}^{m} \neq[0]_{\sim}$ and $[y]_{\sim}^{n} \neq[0]_{\sim}, a n n_{R / \sim}\left([x]_{\sim}[y]_{\sim}\right) \neq$ $\left.\operatorname{ann}_{R / \sim}\left([x]_{\sim}\right) \cup \operatorname{ann}_{R / \sim}\left([y]_{\sim}\right)\right)$. Let $I=[0]_{\sim}$. Then $[x]_{\sim}[y]_{\sim}=[0]_{\sim}$ if and only if $x y \in I$; so $[x]_{\sim}$ and $[y]_{\sim}$ are adjacent in $\Gamma_{\sim}(R)$ (resp., $\left.\bar{\Gamma}_{\sim}(R), A G_{\sim}(R)\right)$ if and only if $x y \in I$ (resp., $x^{m} y^{n} \in I$ for $m, n \in \mathbb{N}$ with $x^{m} \notin I$ and $y^{n} \notin I,(I: x y) \neq(I$ : $x) \cup(I: y)$ ). Note that $Z(R / \sim)^{*}=\emptyset$ if and only if $[0]_{\sim}=R$ (i.e., $\sim=R \times R$ ) or $[0]_{\sim}$ is a prime semigroup ideal of $R([12$, Theorem $3.3(\mathrm{a})])$.

We next give the analogs of Theorems 2.1, 2.8, and 2.11 for congruence-based graphs. Recall that $Z(R / \sim)^{*}=Z(R)^{*}$ for $\sim==_{R}$, and $Z(R / \sim)^{*}=Z_{I}(R)$ for $\sim$ 
given by $x \sim y$ if and only if $x=y$ or $x, y \in I$. Thus, Theorems 2.1, 2.8, and 2.11 are special cases of our next two results.

Theorem 3.1. Let $R$ be a commutative ring with $1 \neq 0, \sim$ a multiplicative congruence relation on $R$, and $I=[0]_{\sim}$.

(a) $\Gamma_{\sim}(R) \subseteq \bar{\Gamma}_{\sim}(R) \subseteq A G_{\sim}(R)$.

(b) $\bar{\Gamma}_{\sim(R)}=\Gamma(R)_{\sim}$ if

(1) $x \in \sqrt{I}$ implies $x^{2} \in I$, and

(2) $x \in R \backslash \sqrt{I}$ implies $\left(I: x^{2}\right)=(I: x)$.

In particular, $\bar{\Gamma}_{\sim}(R)=\Gamma(R)_{\sim}$ when $I$ is a radical semigroup ideal of $R$.

(c) $A G_{\sim}(R)=\Gamma_{\sim}(R)$ if $I$ is a 2-absorbing semigroup ideal of $R$.

Proof. The proof of (a) (resp., (b) and (c)) is similar to that of Theorem 2.1 (resp., Theorem 2.8). Note that (b) and (c) are not "if and only if"; see Example 3.2.

The next example shows that parts (b) and (c) of Theorem 3.1 are not "if and only if" as in Theorem 2.8 .

Example 3.2. Let $R=\mathbb{Z}_{8}$ and $\sim$ be given by $x \sim y \Leftrightarrow \operatorname{ann}_{R}(x)=\operatorname{ann}_{R}(y)$ (equivalently, $x \sim y \Leftrightarrow y=u x$ for some $u \in U(R)$ ). Then $I=[0]=\{0\}$ and $Z(R / \sim)^{*}=\{[2],[4]\} ;$ so $\Gamma_{\sim}(R)=\bar{\Gamma}_{\sim}(R)=A G_{\sim}(R)=K^{2}$. However, $2 \in \sqrt{I}=$ $\{0,2,4,6\}$ and $2^{2}=4 \notin I$; so the converse of Theorem 3.1(b) fails. And $I$ is not a 2-absorbing ideal of $R$; so the converse of Theorem 3.1(c) fails.

Theorem 3.3. Let $R$ be a commutative ring with $1 \neq 0, \sim$ a multiplicative congruence relation on $R$, and $I=[0]_{\sim}$.

(a) $\Gamma_{\sim}(R), \bar{\Gamma}_{\sim}(R)$, and $A G_{\sim}(R)$ are connected.

(b) $0 \leq \operatorname{diam}\left(A G_{\sim}(R)\right) \leq \operatorname{diam}\left(\bar{\Gamma}_{\sim}(R)\right) \leq \operatorname{diam}\left(\Gamma_{\sim}(R)\right) \leq 3$.

(c) $\operatorname{diam}\left(A G_{\sim}(R)\right) \in\{0,1,2\}$.

(d) $\Gamma_{\sim}(R), \bar{\Gamma}_{\sim}(R)$, and $A G_{\sim}(R)$ have girth 3,4 , or $\infty$. Moreover, $\operatorname{gr}\left(A G_{\sim}(R)\right) \leq$ $\operatorname{gr}\left(\bar{\Gamma}_{\sim}(R)\right) \leq \operatorname{gr}\left(\Gamma_{\sim}(R)\right)$.

Proof. The proofs of (a) and (b) follow as those in Theorem 2.11 since $\Gamma_{\sim}(R)$ is connected with $\operatorname{diam}\left(\Gamma_{\sim}(R)\right) \in\{0,1,2,3\} \quad([12$, Theorem 2.7(a) $])$ and $\Gamma_{\sim}(R) \subseteq$ $\bar{\Gamma}_{\sim}(R) \subseteq A G_{\sim}(R)$ by Theorem 3.1(a).

(c) Suppose that distinct vertices $[x]$ and $[y]$ are not adjacent in $A G_{\sim}(R)$. Then $(I: x y)=(I: x) \cup(I: y)$; so we may assume that $(I: y) \subseteq(I: x)=(I: x y)$. Let $w \in(I: y) \backslash I$; so $w \in Z_{I}(R)$ and $w x, w y \in I$. Thus $[x]-[w]-[y]$ is a path of length 2 in $A G_{\sim}(R)$ (actually, in $\Gamma_{\sim}(R)$ ), and hence $\operatorname{diam}\left(A G_{\sim}(R)\right) \leq 2$.

(d) By [12, Theorem 2.7(b)], $g r\left(\Gamma_{\sim}(R)\right) \in\{3,4, \infty\}$. 
First, suppose that $\Gamma_{\sim}(R) \subsetneq \bar{\Gamma}_{\sim}(R)$. By Theorem 3.1(b), either (1) there is an $x \in R$ such that $x^{n} \in I$, but $x^{n-1} \notin I$ for some $n \geq 3$, or (2) there is an $x \in R \backslash \sqrt{I}$ such that $(I: x) \subsetneq\left(I: x^{2}\right)$. If (1) holds and $n \geq 4$, then $[x]-\left[x^{2}\right]-\left[x^{n-1}\right]-[x]$ is a 3 -cycle in $\bar{\Gamma}_{\sim}(R)$. So suppose that $n=3$. If $Z(R / \sim)^{*}=\left\{[x],\left[x^{2}\right]\right\}$, then $\Gamma_{\sim}(R)=\bar{\Gamma}_{\sim}(R)=K^{2}$, a contradiction. Thus by part (a), there is another vertex $[y]$ adjacent to either $[x]$ or $\left[x^{2}\right]$. In either case, $[x]-\left[x^{2}\right]-[y]-[x]$ is a 3 -cycle in $\bar{\Gamma}_{\sim}(R)$. If (2) holds, let $y \in R$ with $y x^{2} \in I$, but $y x \notin I$. If $y^{2} \in I$, then $[y]-[x]-[x y]-[y]$ is a cycle; and if $y^{2} \notin I$, then $[x]-[x y]-\left[x^{2}\right]-[y]-[x]$ is a cycle. Hence $\operatorname{gr}\left(\bar{\Gamma}_{\sim}(R)\right) \in\{3,4, \infty\}$.

Next, suppose that $\Gamma_{\sim}(R) \subsetneq A G_{\sim}(R)$. Let $[x]$ and $[y]$ be adjacent in $A G_{\sim}(R)$, but not in $\Gamma_{\sim}(R)$. Then $(I: x y) \neq(I: x) \cup(I: y)$ and $x y \notin I$; so there is a $z \in Z_{I}(R)$ with $z x y \in I$, but $z x, z y \notin I$. If $[z] \notin\{[x],[y]\}$, then $[x]-[z]-[y]-[x]$ is a 3 -cycle in $A G_{\sim}(R)$. If, say, $[z]=[x]$, then $[x]-[y]-\left[x^{2}\right]-[x y]-[x]$ is a cycle and $[y] \neq[x],[y] \neq[x y] \neq[x]$. Thus $\operatorname{gr}\left(A G_{\sim}(R)\right) \in\{3,4, \infty\}$.

The "moreover" statement is clear.

Let $G$ be a simple graph. We say that distinct adjacent vertices $x$ and $y$ of $G$ are orthogonal, written $x \perp y$, if there is no vertex $z$ of $G$ adjacent to both $x$ and $y$ (i.e., the edge $x-y$ is not part of a triangle in $G$ ). The graph $G$ is complemented if for every vertex $x$ of $G$, there is a vertex $y$ of $G$ with $x \perp y$ ( $y$ is called a complement of $x$ ), and $G$ is uniquely complemented if $G$ is complemented and whenever $x \perp y$ and $x \perp z$ for vertices $x, y, z$ of $G$, then $N(y)=N(z)$. Note that for distinct $[x]_{\sim}$ and $[y]_{\sim}$ in $\Gamma_{\sim}(R)$, we have $[x]_{\sim} \perp[y]_{\sim}$ if and only if $x y \in I$ and $(I: x) \cap(I: y) \subseteq I \cup[x]_{\sim} \cup[y]_{\sim}$, where $I=[0]_{\sim}$.

In [11], it was determined when $\Gamma(R)$ is complemented or uniquely complemented. In particular, $\Gamma(R)$ is uniquely complemented if and only if $T(R)$ is von Neumann regular or $\Gamma(R)$ is a star graph ([11, Corollary 3.10]); $\Gamma(R)$ is complemented, but not uniquely complemented, if and only if $R \cong D \times B$, where $D$ is an integral domain and $B$ is $\mathbb{Z}_{4}$ or $\mathbb{Z}_{2}[X] /\left(X^{2}\right)$ ([11, Theorem 3.14]); and if $R$ is reduced, then $\Gamma(R)$ is uniquely complemented if and only if $\Gamma(R)$ is complemented, if and only if $T(R)$ is von Neumann regular ([11, Theorem 3.5]). This was generalized to $\Gamma_{I}(R)$ in [32]. In particular, if $I$ is nonzero, then $\Gamma_{I}(R)$ is uniquely complemented if and only if $\Gamma_{I}(R)$ is complemented; and if $I$ is a radical ideal of $R$, then $\Gamma_{I}(R)$ is complemented if and only if $\Gamma(R / I)$ is complemented ([32, Theorem 2.10]). We extend these results to $\Gamma_{\sim}(R)$.

We will need the following lemma. Note that $[x]_{\sim}=[y]_{\sim}$ does not imply that $x+I=y+I$, or conversely. 
Lemma 3.4. Let $R$ be a commutative ring with $1 \neq 0, \sim$ a multiplicative congruence relation on $R$ with $I=[0]_{\sim}$ a radical ideal of $R$, and $x, y \in R$. Then $[x]_{\sim}$ and $[y]_{\sim}$ are distinct adjacent vertices in $\Gamma_{\sim}(R)$ if and only $x+I$ and $y+I$ are distinct adjacent vertices in $\Gamma(R / I)$. Moreover, $\Gamma_{\sim}(R)$ is complemented if and only if $\Gamma(R / I)$ is complemented.

Proof. Suppose that $[x]_{\sim}$ and $[y]_{\sim}$ are distinct adjacent vertices in $\Gamma_{\sim}(R)$. Then $x y \in I$. If $x+I=y+I$, then $x-y \in I$, and thus $x^{2}=x(x-y)+x y \in I$. Hence $x \in I$ since $I$ is a radical ideal of $R$. Thus $[x]_{\sim}=[0]_{\sim}$, a contradiction. Hence $x+I$ and $y+I$ are distinct adjacent vertices in $\Gamma(R / I)$.

Conversely, suppose that $x+I$ and $y+I$ are distinct adjacent vertices in $\Gamma(R / I)$. Then $x y \in I$. If $[x]_{\sim}=[y]_{\sim}$, then $x \sim y$, and thus $x^{2} \sim x y \in I=[0]_{\sim}$. Hence $x^{2} \in I$, and thus $x \in I$ since $I$ is a radical ideal of $R$. Hence $x+I=I$, a contradiction. Thus $[x]_{\sim}$ and $[y]_{\sim}$ are distinct adjacent vertices in $\Gamma_{\sim}(R)$.

The "moreover" statement is clear.

Theorem 3.5. Let $R$ be a commutative ring with $1 \neq 0, \sim$ a multiplicative congruence relation on $R$, and $I=[0]_{\sim}$.

(a) If I is a radical semigroup ideal of $R$, then $\Gamma_{\sim}(R)$ is uniquely complemented if and only if $\Gamma_{\sim}(R)$ is complemented.

(b) If $I$ is a radical ideal of $R$, then the following statements are equivalent.

(1) $\Gamma_{\sim}(R)$ is uniquely complemented.

(2) $\Gamma_{\sim}(R)$ is complemented.

(3) $\Gamma(R / I)$ is uniquely complemented.

(4) $\Gamma(R / I)$ is complemented.

(5) $T(R / I)$ is von Neumann regular.

Proof. (a) Clearly $\Gamma_{\sim}(R)$ is complemented if $\Gamma_{\sim}(R)$ is uniquely complemented. So suppose that $\Gamma_{\sim}(R)$ is complemented. Let $[x] \perp[y]$ and $[x] \perp[z]$ for distinct vertices $[x],[y],[z]$ of $\Gamma_{\sim}(R)$ (we omit the " $\sim$ " on each $[a] \sim$ ); we show that $N([y])=N([z])$. Let $[w] \in N([y])$; so $[w][y]=[0]$. If $[w][z] \neq[0]$, then $[v]=[w][z]$ is adjacent to both $[x]$ and $[y]$. This follows since $[v][x]=([w][z])[x]=[w]([x][z])=[w][0]=[0]$ and $[v][y]=([w][z])[y]=([w][y])[z]=[0][z]=[0]$. Also, $[v] \neq[x]$ since $[v]=[x]$ implies $[x]^{2}=[v][x]=[0]$, and thus $x \in I$ since $I$ is a radical semigroup ideal of $R$. But then $[x]=[0]$, a contradiction. Similarly, $[v]=[y]$ implies $[y]^{2}=[v][y]=$ $([w][z])[y]=([w][y])[z]=[0][z]=[0]$, and hence $[y]=[0]$. So $[x],[y]$, and $[v]$ are all distinct. This contradicts that $[x] \perp[y]$. Thus $[w][z]=[0]$, and $[w] \neq[z]$ since 
$[x] \perp[z]$. Hence $[w] \in N([z])$; so $N([y]) \subseteq N([z])$. Similarly, $N([z]) \subseteq N([y])$; so $N([y])=N([z])$. Thus $\Gamma_{\sim}(R)$ is uniquely complemented.

(b) (1) and (2) are equivalent by part (a). The equivalence of (2) and (4) follows from Lemma 3.4. The equivalence of (3) - (5) follows from [11, Theorem 3.5] since $R / I$ is reduced.

Corollary 3.6. ([32, Theorem 2.10]) Let $R$ be a commutative ring and I a radical ideal of $R$. Then the following statements are equivalent.

(1) $\Gamma_{I}(R)$ is uniquely complemented.

(2) $\Gamma_{I}(R)$ is complemented.

(3) $\Gamma(R / I)$ is uniquely complemented.

(4) $\Gamma(R / I)$ is complemented.

(5) $T(R / I)$ is von Neumann regular.

\section{Compressed graphs}

In this section, we study "compressed" graphs. The compressed zero-divisor graph $\Gamma_{E}(R)$ (using different notation) was first defined by S. B. Mulay ([29, p. 3551]). Specifically, let $\sim$ be the multiplicative congruence relation on $R$ defined by $x \sim y \Leftrightarrow \operatorname{ann}_{R}(x)=a n n_{R}(y)$. Then $\Gamma_{E}(R)=\Gamma_{\sim}(R)$ with vertices $Z(R / \sim)^{*}$, and distinct vertices $[x]$ and $[y]$ are adjacent if and only if $[x][y]=[0]$, if and only if $x y=0$, since $[0]=\{0\}$. Analogously, we define $\bar{\Gamma}_{E}(R)=\bar{\Gamma}_{\sim}(R)$, and $A G_{E}(R)=A G_{\sim}(R)$. Note that $\Gamma_{E}(R) \subseteq \bar{\Gamma}_{E}(R) \subseteq A G_{E}(R)$ by Theorem 3.1(a). The compressed annihilator graph $A G_{E}(R)$ was introduced in [30]. Also, see [8], [10], [21], and [33] for more on $\Gamma_{E}(R)$.

Let $G$ be a simple graph. For $x, y \in V(G)$, define $x \equiv y \Leftrightarrow N_{G}(x) \backslash\{y\}=$ $N_{G}(y) \backslash\{x\}$. Then $\equiv$ is an equivalence relation on $V(G)$ ([10, Theorem 2.1]). Let $G / \equiv$ be the (simple) graph with vertices $V(G) / \equiv=\{[x] \mid x \in G\}$, and distinct vertices $[x]$ and $[y]$ are adjacent if and only if some (and hence every) element of $[x]$ is adjacent to some (and hence every) element of $[y]$. Note that for $G=\Gamma(R)$, $x \equiv y$ in $\Gamma(R)$ if and only if $a n n_{R}(x) \backslash\{x, y\}=a n n_{R}(y) \backslash\{x, y\}$. Moreover, if $R \neq \mathbb{Z}_{2} \times \mathbb{Z}_{2}$, then $\Gamma_{E}(R) \cong \Gamma(R) / \equiv\left(\left[10\right.\right.$, Theorem 2.5]). Thus, the graph $\Gamma_{E}(R)$ is completely determined by, and may be computed from, $\Gamma(R)$ when $R \neq \mathbb{Z}_{2} \times \mathbb{Z}_{2}$. In particular, if $|\Gamma(R)|>2$, then $\Gamma_{E}(R) \cong \Gamma_{E}(S)$ if and only if $\Gamma(R) \cong \Gamma(S)$. However, for $R=\mathbb{Z}_{2} \times \mathbb{Z}_{2}$ and $S=\mathbb{Z}_{3}[X] /\left(X^{2}\right)$, we have $\Gamma(R)=\Gamma(S)=K^{2}$, but $\Gamma_{E}(R)=K^{2}$ and $\Gamma_{E}(S)=K^{1}$.

The compressed zero-divisor graph $\Gamma_{E}(R)$ "compresses" $\Gamma(R)$ by identifying vertices with the same adjacency relations. However, $\bar{\Gamma}_{E}(R)$ and $A G_{E}(R)$ need not 
"compress" $\bar{\Gamma}(R)$ and $A G(R)$ in this way (see Example 4.2). We next define the "compressed" graphs associated to $\bar{\Gamma}(R)$ and $A G(R)$. Although all the graphs we have studied in this paper are simple graphs, it is sometimes convenient to consider these graphs to have loops. For example, there is a loop at $x \in Z(R)^{*}$ in $\Gamma(R)$ (resp., $\bar{\Gamma}(R), A G(R))$ if and only if $x^{2}=0$ (resp., $\left.x \in \operatorname{nil}(R), a n n_{R}\left(x^{2}\right) \neq a n n_{R}(x)\right)$. We will denote the graph $\Gamma(R)$ (resp., $\bar{\Gamma}(R), A G(R)$ ) with loops added by $\Gamma^{L}(R)$ (resp., $\left.\bar{\Gamma}^{L}(R), A G^{L}(R)\right)$.

Let $G$ be $\Gamma(R), \bar{\Gamma}(R)$, or $A G(R)$. For $x \in Z(R)^{*}$, define their extended edge sets $E_{G}^{*}(x)$ to be $N_{G}(x) \cup\{x \mid$ if $x$ is a loop in $G\}$; so $N_{G}(x) \subseteq E_{G}^{*}(x) \subseteq \bar{N}_{G}(x)$. Thus $E_{\Gamma(R)}^{*}(x)=\left\{y \in Z(R)^{*} \mid x y=0\right\}, E_{\bar{\Gamma}(R)}^{*}(x)=\left\{y \in Z(R)^{*} \mid x^{m} y^{n}=0\right.$ for $m, n \in \mathbb{N}$ with $\left.x^{m} \neq 0, y^{n} \neq 0\right\}$, and $E_{A G(R)}^{*}(x)=\left\{y \in Z(R)^{*} \mid a n n_{R}(x y) \neq\right.$ $\left.\operatorname{ann}_{R}(x) \cup \operatorname{ann}_{R}(y)\right\}$. In particular, $x \in E_{\Gamma(R)}^{*}(x) \Leftrightarrow x^{2}=0, x \in E_{\bar{\Gamma}(R)}^{*}(x) \Leftrightarrow$ $x \in \operatorname{nil}(R)$, and $x \in E_{A G(R)}^{*}(x) \Leftrightarrow \operatorname{ann}_{R}\left(x^{2}\right) \neq a n n_{R}(x)$.

For the graphs $G=\Gamma(R), \bar{\Gamma}(R)$, and $A G(R)$, the relation $\sim_{G}$ defined by $x \sim_{G} y$ $\Leftrightarrow E_{G}^{*}(x)=E_{G}^{*}(y)$ is an equivalence relation on $V(G)=Z(R)^{*}$. We define the $\sim_{G}$-compressed graph of $G$ to be the simple graph $G / \sim_{G}$ with vertices $V\left(G / \sim_{G}\right)$ $=V(G) / \sim_{G}=\left\{[x]_{\sim_{G}} \mid x \in V(G)\right\}$, and distinct vertices $[x]_{\sim_{G}}$ and $[y]_{\sim_{G}}$ are adjacent if and only if $x$ and $y$ are adjacent in $G$. (This graph is well-defined since if $x \sim_{G} a$ and $y \sim_{G} b$, then $x$ and $y$ are adjacent if and only if $a$ and $b$ are adjacent.) We denote the associated $\sim_{G}$-compressed graphs by $C \Gamma(R), C \bar{\Gamma}(R)$, and $C A G(R)$, respectively. In the obvious way, one can also define $C \Gamma_{\sim}(R), C \bar{\Gamma}_{\sim}(R)$, and $C A G_{\sim}(R)$ for $\sim$ any multiplicative congruence relation on $R$; details are left to the interested reader.

The equivalence relation $\sim_{G}$ on $Z(R)^{*}$ may be extended to an equivalence relation on $R$ by defining $[0]_{\sim_{G}}=\{0\}$ and $[1]_{\sim_{G}}=R \backslash Z(R)$. We will see that $\sim_{\Gamma(R)}$ is actually a multiplicative congruence relation on $R$. However, the following example shows that $\sim_{G}$ need not be a multiplicative congruence relation on $R\left(\right.$ or $Z(R)^{*}$ ) when $G$ is $\bar{\Gamma}(R)$ or $A G(R)$.

Example 4.1. Let $R=\mathbb{Z}_{2} \times \mathbb{Z}_{8}$.

(a) Then $R / \sim_{\Gamma(R)}=\{[(0,0)],[(1,1)],[(0,1)],[(0,2)],[(0,4)],[(1,0)],[(1,2)],[(1,4)]\}$, where $[(0,0)]=\{(0,0)\},[(1,1)]=\{(1,1),(1,3),(1,5),(1,7)\},[(0,1)]=\{(0,1)$, $(0,3),(0,5),(0,7)\},[(0,2)]=\{(0,2),(0,6)\},[(0,4)]=\{(0,4)\},[(1,0)]=\{(1,0)\}$, $[(1,2)]=\{(1,2),(1,6)\}$, and $[(1,4)]=\{(1,4)\}$. Note that $\sim_{\Gamma(R)}$ is a multiplicative congruence relation on $R$ (and $\left.Z(R)^{*}\right)$, and $V(C \Gamma(R))=\{[(0,1)],[(0,2)],[(0,4)]$, $[(1,0)],[(1,2)],[(1,4)]\}$. 
(b) Then $R / \sim_{\bar{\Gamma}(R)}=\{[(0,0)],[(1,1)],[(0,1)],[(0,2)],[(1,0)]\}$, where $[(0,0)]=\{(0,0)\},[(1,1)]=\{(1,1),(1,3),(1,5),(1,7)\},[(0,1)]=\{(0,1),(0,3)$, $(0,5),(0,7)\},[(0,2)]=\{(0,2),(0,4),(0,6)\}$, and $[(1,0)]=\{(1,0),(1,2),(1,4),(1,6)\}$. We have $(0,2) \sim_{\bar{\Gamma}(R)}(0,4)$, but $(0,2)(0,2)=(0,4) \chi_{\bar{\Gamma}(R)}(0,0)=(0,2)(0,4)$. Thus $\sim_{\bar{\Gamma}(R)}$ is not a multiplicative congruence relation on $R\left(\right.$ or $\left.Z(R)^{*}\right)$, and $V(C \bar{\Gamma}(R))=\{[(0,1)],[(0,2)],[(1,0)]\}$.

(c) Then $R / \sim_{A G(R)}=\{[(0,0)],[(1,1)],[(0,1)],[(0,2)],[(1,0),[(1,2)]\}$, where $[(0,0)]=\{(0,0)\},[(1,1)]=\{(1,1),(1,3),(1,5),(1,7)\},[(0,1)]=\{(0,1),(0,3)$, $(0,5),(0,7)\},[(0,2)]=\{(0,2),(0,4),(0,6)\},[(1,0)]=\{(1,0)\}$, and $[(1,2)]=\{(1,2)$, $(1,4),(1,6)\}$. We have $(1,2) \sim_{A G(R)}(1,4)$, but $(1,2)(1,2)=(1,4) \varkappa_{A G(R)}(1,0)=$ $(1,2)(1,4)$. Thus $\sim_{A G(R)}$ is not a multiplicative congruence relation on $R$ (or $\left.Z(R)^{*}\right)$, and $V(C A G(R))=\{[(0,1)],[(0,2)],[(1,0),[(1,2)]\}$.

For $G=\Gamma(R)$ and $x \in Z(R)^{*}, E_{\Gamma(R)}^{*}(x)=\operatorname{ann}_{R}(x)^{*}$. Thus $x \sim_{\Gamma(R)} y \Leftrightarrow$ $\operatorname{ann}_{R}(x)=\operatorname{ann}_{R}(y)$; so $\sim_{\Gamma(R)}$ is a multiplicative congruence relation and $C \Gamma(R)=$ $\Gamma_{E}(R)$. By definition, $[x]_{\sim_{\bar{\Gamma}(R)}}$ and $[y]_{\sim_{\bar{\Gamma}(R)}}$ are adjacent in $C \bar{\Gamma}(R)$ if and only if $x^{m} y^{n}=0$ for positive integers $m$ and $n$ with $x^{m} \neq 0$ and $y^{n} \neq 0$, and $[x]_{\sim_{A G(R)}}$ and $[y]_{\sim_{A G(R)}}$ are adjacent in $C A G(R)$ if and only if $\operatorname{ann}_{R}(x y) \neq a n n_{R}(x) \cup a n n_{R}(y)$.

Although $C \Gamma(R)=\Gamma_{E}(R)$, we need not have $C \bar{\Gamma}(R)=\bar{\Gamma}_{E}(R)$ or $C A G(R)=$ $A G_{E}(R)$. For example, this is the case when $R=\mathbb{Z}_{2} \times \mathbb{Z}_{8}$. Below are the graphs for $\Gamma(R), \bar{\Gamma}(R), A G(R), \Gamma_{E}(R)=C \Gamma(R), \bar{\Gamma}_{E}(R), A G_{E}(R), C \bar{\Gamma}(R)$, and $C A G(R)$ for $R=\mathbb{Z}_{2} \times \mathbb{Z}_{8}$ (cf., Example 4.1).

Example 4.2. The graphs for $\Gamma(R), \bar{\Gamma}(R), A G(R), \Gamma_{E}(R)=C \Gamma(R), \bar{\Gamma}_{E}(R), A G_{E}(R)$, $C \bar{\Gamma}(R)$, and $C A G(R)$ for $R=\mathbb{Z}_{2} \times \mathbb{Z}_{8}$ (cf., Example 4.1).

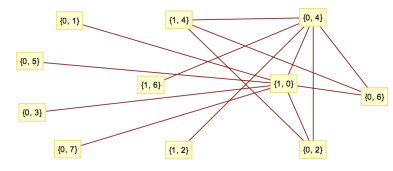

$\Gamma\left(\mathbb{Z}_{2} \times \mathbb{Z}_{8}\right)$

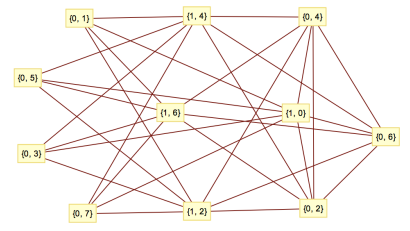

$\bar{\Gamma}\left(\mathbb{Z}_{2} \times \mathbb{Z}_{8}\right)$

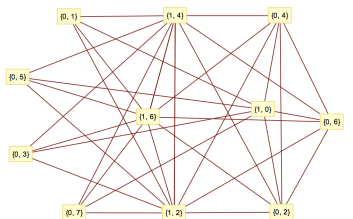

$A G\left(\mathbb{Z}_{2} \times \mathbb{Z}_{8}\right)$ 


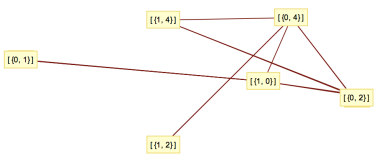

$\Gamma_{E}\left(\mathbb{Z}_{2} \times \mathbb{Z}_{8}\right)$

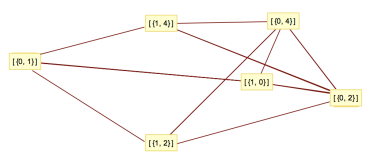

$\bar{\Gamma}_{E}\left(\mathbb{Z}_{2} \times \mathbb{Z}_{8}\right)$

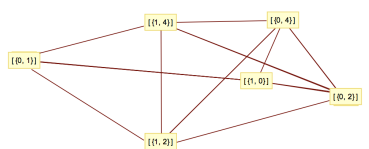

$A G_{E}\left(\mathbb{Z}_{2} \times \mathbb{Z}_{8}\right)$

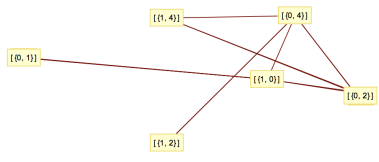

$C \Gamma\left(\mathbb{Z}_{2} \times \mathbb{Z}_{8}\right)$

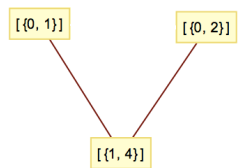

$C \bar{\Gamma}\left(\mathbb{Z}_{2} \times \mathbb{Z}_{8}\right)$

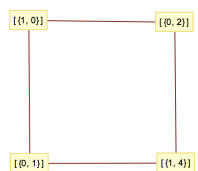

$C A G\left(\mathbb{Z}_{2} \times \mathbb{Z}_{8}\right)$

We next consider some cases when $C \bar{\Gamma}(R)=\bar{\Gamma}_{E}(R)$ and $C A G(R)=A G_{E}(R)$. Although the next result may seem obvious, note that we need to consider loops.

Theorem 4.3. Let $R$ be a commutative ring with $1 \neq 0$.

(a) If $\bar{\Gamma}(R)=\Gamma(R)$, then $C \bar{\Gamma}(R)=\bar{\Gamma}_{E}(R)=\Gamma_{E}(R)$.

(b) If $A G(R)=\Gamma(R)$, then $C A G(R)=A G_{E}(R)=\Gamma_{E}(R)$.

(c) If $C \bar{\Gamma}(R)=\Gamma_{E}(R)$, then $\bar{\Gamma}(R)=\Gamma(R)$.

Proof. (a) Suppose that $\bar{\Gamma}(R)=\Gamma(R)$. Clearly $\bar{\Gamma}_{E}(R)=\Gamma_{E}(R)$. To show that $C \bar{\Gamma}(R)=\bar{\Gamma}_{E}(R)$, we need only show that $E_{\bar{\Gamma}(R)}^{*}(x)=E_{\Gamma(R)}^{*}(x)$ for every $x \in Z(R)^{*}$ since $C \bar{\Gamma}(R)$ and $\bar{\Gamma}_{E}(R)$ have the same adjacency relations. Since $\bar{\Gamma}(R)=\Gamma(R)$ by hypothesis, $E_{\bar{\Gamma}(R)}^{*}(x) \backslash\{x\}=E_{\Gamma(R)}^{*}(x) \backslash\{x\}$ for every $x \in Z(R)^{*}$. So we need only show that $x^{2}=0 \Leftrightarrow x \in \operatorname{nil}(R)$. The $(\Rightarrow)$ implication is clear; so suppose by way of contradiction that $x^{n}=0$, but $x^{n-1} \neq 0$, for some integer $n \geq 3$. Let $z=x\left(1+x^{n-2}\right) \in Z(R)^{*}$. Then $z \neq x$ and $z \in\left(E_{\bar{\Gamma}(R)}^{*}(x) \backslash\{x\}\right) \backslash\left(E_{\Gamma(R)}^{*}(x) \backslash\{x\}\right)$, a contradiction. Thus $(\Leftarrow)$ also holds; so $E_{\bar{\Gamma}(R)}^{*}(x)=E_{\Gamma(R)}^{*}(x)$. Hence $C \bar{\Gamma}(R)=$ $\bar{\Gamma}_{E}(R)$.

(b) Suppose that $A G(R)=\Gamma(R)$. Clearly $A G_{E}(R)=\Gamma_{E}(R)$. To show that $C A G(R)=A G_{E}(R)$, we need only show that $E_{A G(R)}^{*}(x)=E_{\Gamma(R)}^{*}(x)$ for every $x \in Z(R)^{*}$ since $C A G(R)$ and $A G_{E}(R)$ have the same adjacency relations. Since $A G(R)=\Gamma(R)$ by hypothesis, $E_{A G(R)}^{*}(x) \backslash\{x\}=E_{\Gamma(R)}^{*}(x) \backslash\{x\}$ for every $x \in$ $Z(R)^{*}$. So we need only show that $x^{2}=0 \Leftrightarrow a n n_{R}\left(x^{2}\right) \neq a n n_{R}(x)$. The $(\Rightarrow)$ 
implication is clear; so suppose by way of contradiction that $a n n_{R}\left(x^{2}\right) \neq a n n_{R}(x)$, but $x^{2} \neq 0$. Let $y \in \operatorname{ann}_{R}\left(x^{2}\right) \backslash a n n_{R}(x)$; so $y x^{2}=0$ and $y x \neq 0$. If $y \neq x$, then $y \in\left(E_{A G(R)}^{*}(x) \backslash\{x\}\right) \backslash\left(E_{\Gamma(R)}^{*}(x) \backslash\{x\}\right)$ since $\operatorname{ann}_{R}(x y) \neq \operatorname{ann}_{R}(x) \cup \operatorname{ann}_{R}(y)$, a contradiction. If $y=x$, then $x^{3}=0$ and $x^{2} \neq 0$. Let $z=x(1+x) \in Z(R)^{*}$. Then $z \neq x, z x \neq 0$, and $z x^{2}=0$. Hence $z \in\left(E_{A G(R)}^{*}(x) \backslash\{x\}\right) \backslash\left(E_{\Gamma(R)}^{*}(x) \backslash\{x\}\right)$ since $\operatorname{ann}_{R}(x z) \neq a n n_{R}(x) \cup a n n_{R}(z)$, a contradiction. Thus $x^{2}=0$; so $(\Leftarrow)$ also holds and $E_{A G(R)}^{*}(x)=E_{\Gamma(R)}^{*}(x)$. Hence $C A G(R)=A G_{E}(R)$.

(c) Suppose that $C \bar{\Gamma}(R)=\Gamma_{E}(R)$. By Corollary 2.9(1), to show that $\bar{\Gamma}(R)=$ $\Gamma(R)$ we need to show that $(1) x \in \operatorname{nil}(R)$ implies $x^{2}=0$ and (2) $x \in R \backslash \operatorname{nil}(R)$ implies $\operatorname{ann}_{R}\left(x^{2}\right)=a n n_{R}(x)$. For (1), assume by way of contradiction that $x^{n}=$ 0 , but $x^{n-1} \neq 0$, for some integer $n \geq 3$. Then $[x]_{\sim_{\Gamma(R)}}$ and $\left[x^{n-1}\right]_{\sim_{\Gamma(R)}}$ are distinct adjacent vertices in $\Gamma_{E}(R)$. However, $[x]_{\sim_{\bar{\Gamma}(R)}}=\left[x^{n-1}\right]_{\sim_{\bar{\Gamma}(R)}}$ in $C \bar{\Gamma}(R)$ since $E_{\bar{\Gamma}(R)}^{*}(x)=E_{\bar{\Gamma}(R)}^{*}\left(x^{n-1}\right)$. This contradicts $C \bar{\Gamma}(R)=\Gamma_{E}(R)$; so (1) holds. For (2), suppose by way of contradiction that $a n n_{R}\left(x^{2}\right) \neq a n n_{R}(x)$. Let $y \in R$ with $y x^{2}=0$ and $y x \neq 0$. Then $[x]_{\sim_{\Gamma(R)}}$ and $\left[x^{2}\right]_{\sim_{\Gamma(R)}}$ are distinct adjacent vertices in

$\Gamma_{E}(R)$. However, $[x]_{\sim_{\bar{\Gamma}(R)}}=\left[x^{2}\right]_{\sim_{\bar{\Gamma}(R)}}$ in $C \bar{\Gamma}(R)$ since $E_{\bar{\Gamma}(R)}^{*}(x)=E_{\bar{\Gamma}(R)}^{*}\left(x^{2}\right)$. This contradicts $C \bar{\Gamma}(R)=\Gamma_{E}(R)$; so (2) holds. Thus $\bar{\Gamma}(R)=\Gamma(R)$.

Remark 4.4. (a) The converse of Theorem 4.3(b) does not hold for $R=\mathbb{Z}_{30}$ since $C A G\left(\mathbb{Z}_{30}\right)=A G_{E}\left(\mathbb{Z}_{30}\right)$, but $\Gamma\left(\mathbb{Z}_{30}\right) \subsetneq A G\left(\mathbb{Z}_{30}\right)$ since 2 and 3 are adjacent in $A G\left(\mathbb{Z}_{30}\right)$, but not in $\Gamma\left(\mathbb{Z}_{30}\right)$.

(b) The proof of Theorem 4.3 shows that $\Gamma(R)=\bar{\Gamma}(R)$ implies $\Gamma^{L}(R)=\bar{\Gamma}^{L}(R)$ and $\Gamma(R)=A G(R)$ implies $\Gamma^{L}(R)=A G^{L}(R)$. However, for $R=\mathbb{Z}_{2} \times \mathbb{Z}_{4}$, we have $\bar{\Gamma}(R)=A G(R)$, but $\bar{\Gamma}^{L}(R) \subsetneq A G^{L}(R)$ since there is a loop at $(1,2)$ in $A G^{L}(R)$, but not in $\bar{\Gamma}^{L}(R)$.

(c) (cf. Example 4.2) Let $R=\mathbb{Z}_{p_{1} n_{1}} \times \cdots \times \mathbb{Z}_{p_{k} n_{k}}$, where the $p_{i}$ are (not necessarily distinct) primes and every $n_{i} \in \mathbb{N}$. If every $n_{i} \leq 2$, then $C \bar{\Gamma}(R)=\bar{\Gamma}_{E}(R)$ and $C A G(R)=A G_{E}(R)$, otherwise $C \bar{\Gamma}(R) \neq \bar{\Gamma}_{E}(R)$ and $C A G(R) \neq A G_{E}(R)$ (cf. [28, Cororally 2.8.1 and Proposition 2.16]).

\section{Maps between graphs}

In this section, we study maps between congruence-based graphs. This extends the work in [12] on $\Gamma_{\sim}(R)$ to $\bar{\Gamma}_{\sim}(R)$ and $A G_{\sim}(R)$.

First, we recall several results from [12]. For a commutative $\operatorname{ring} R$, let $\mathcal{C}(R)$ be the set of multiplicative congruence relations on $R$. We can partially order $\mathcal{C}(R)$ by $\sim_{1} \leq \sim_{2} \Leftrightarrow \sim_{1} \subseteq \sim_{2}$ (as subsets of $R \times R$ ). So $\mathcal{C}(R)$ has a least element $=_{R}=\{(x, x) \mid x \in R\}$ and a greatest element $R \times R$. For $\sim \in \mathcal{C}(R),[0]_{\sim}$ is a 
semigroup ideal of $R$, and moreover, $\mathcal{C}(R)=\sqcup_{I} \mathcal{C}_{I}(R)$, where $I$ is a semigroup ideal of $R$ and $\mathcal{C}_{I}(R)=\left\{\sim \in \mathcal{C}(R) \mid[0]_{\sim}=I\right\}$.

Let $I$ be a semigroup ideal of $R$ and $\sim_{1}, \sim_{2} \in \mathcal{C}(R)$ with $\sim_{1} \leq \sim_{2}$. Then it is easily verified that $1_{R}: R \longrightarrow R$ induces a surjective monoid homomorphism $f: R / \sim_{1} \longrightarrow R / \sim_{2}$ given by $f\left([x]_{\sim_{1}}\right)=[x]_{\sim_{2}}$ with $f\left([0]_{\sim_{1}}\right)=[0]_{\sim_{2}}$ and $f\left([1]_{\sim_{1}}\right)=$ $[1]_{\sim_{2}}$. In fact, $f$ is well-defined if and only if $\sim_{1} \leq \sim_{2}$, and $f$ is injective if and only if $\sim_{1}=\sim_{2}$. Now suppose, in addition, that $\sim_{1}, \sim_{2} \in \mathcal{C}_{I}(R)$ (i.e., $I=[0]_{\sim_{1}}=[0]_{\sim_{2}}$ ). Then $[x]_{\sim_{1}} \in Z\left(R / \sim_{1}\right)^{*}$ if and only if $[x]_{\sim_{2}} \in Z\left(R / \sim_{2}\right)^{*}([12$, Lemma 2.4]). Thus $f$ induces a surjective function $F: \Gamma_{\sim_{1}}(R) \longrightarrow \Gamma_{\sim_{2}}(R)$ given by $F\left([x]_{\sim_{1}}\right)=[x]_{\sim_{2}}$, i.e., $F=\left.f\right|_{Z\left(R / \sim_{1}\right)^{*}}$. Moreover, for distinct adjacent vertices $[x]_{\sim_{1}}$ and $[y]_{\sim_{1}}$ in $\Gamma_{\sim_{1}}(R)$, either $F\left([x]_{\sim_{1}}\right)=F\left([y]_{\sim_{1}}\right)$ or $F\left([x]_{\sim_{1}}\right)$ and $F\left([y]_{\sim_{1}}\right)$ are adjacent in $\Gamma_{\sim_{2}}(R)$. Note that $F$ may be well-defined or injective (and hence a graph isomorphism) without $f$ being well-defined or injective (cf.[12, Remark 2.6(c)]).

Since $F$ is surjective, there is an (not necessarily unique) injective function $G: \Gamma_{\sim_{2}}(R) \longrightarrow \Gamma_{\sim_{1}}(R)$ such that $F G=1_{\Gamma_{\sim_{2}}(R)}$ (i.e., for each $z \in Z\left(R / \sim_{2}\right)^{*}$, choose an $\alpha(z) \in Z\left(R / \sim_{1}\right)^{*}$ such that $F(\alpha(z))=z$, and then define $G(z)=\alpha(z)$ ). Moreover, $G\left([x]_{\sim_{2}}\right)$ and $G\left([y]_{\sim_{2}}\right)$ are adjacent in $\Gamma_{\sim_{1}}(R)$ if and only if $[x]_{\sim_{2}}$ and $[y]_{\sim_{2}}$ are adjacent in $\Gamma_{\sim_{2}}(R)$ since $[0]_{\sim_{1}}=[0]_{\sim_{2}}=I$. Thus $G$ is an injective graph homomorphism and embeds $\Gamma_{\sim_{2}}(R)$ as an induced subgraph of $\Gamma_{\sim_{1}}(R)$. We record these observations in the following theorem.

Theorem 5.1. ([12, Theorem 3.1]) Let $R$ be a commutative ring with $1 \neq 0$, and let $\sim_{1}, \sim_{2} \in \mathcal{C}(R)$ with $\sim_{1} \leq \sim_{2}$. Then there is a surjective monoid homomorphism $f: R / \sim_{1} \longrightarrow R / \sim_{2}$ given by $f\left([x]_{\sim_{1}}\right)=[x]_{\sim_{2}}$. If $[0]_{\sim_{1}}=[0]_{\sim_{2}}$, then $f$ induces a surjective function $F: \Gamma_{\sim_{1}}(R) \longrightarrow \Gamma_{\sim_{2}}(R)$ given by $F\left([x]_{\sim_{1}}\right)=[x]_{\sim_{2}}$ and an injective graph homomorphism $G: \Gamma_{\sim_{2}}(R) \longrightarrow \Gamma_{\sim_{1}}(R)$ such that $F G=1_{\Gamma_{\sim_{2}}(R)}$. Moreover, for adjacent vertices $[x]_{\sim_{1}}$ and $[y]_{\sim_{1}}$ in $\Gamma_{\sim_{1}}(R)$, either $F\left([x]_{\sim_{1}}\right)=F\left([y]_{\sim_{1}}\right)$ or $F\left([x]_{\sim_{1}}\right)$ and $F\left([y]_{\sim_{1}}\right)$ are adjacent in $\Gamma_{\sim_{2}}(R)$; and $\Gamma_{\sim_{2}}(R)$ is isomorphic to an induced subgraph of $\Gamma_{\sim_{1}}(R)$.

Since all three congruence-based graphs have the same vertex set $Z(R / \sim)^{*}=$ $Z(R / \sim) \backslash\left\{[0]_{\sim}\right\}$, these results extend in the natural way to $\bar{\Gamma}_{\sim}(R)$ and $A G_{\sim}(R)$. We need only check adjacency. Note that the functions $F, F^{\prime}, F^{\prime \prime}$ (resp., $G, G^{\prime}, G^{\prime \prime}$ ) take the same values on $Z\left(R / \sim_{1}\right)^{*}$ (resp., $\left.Z\left(R / \sim_{2}\right)^{*}\right)$, but to avoid any possible confusion on which graphs are being considered, we will use the " 's". 
Theorem 5.2. Let $R$ be a commutative ring with $1 \neq 0$, and let $\sim_{1}, \sim_{2} \in$ $\mathcal{C}(R)$ with $\sim_{1} \leq \sim_{2}$. If $[0]_{\sim_{1}}=[0]_{\sim_{2}}$, then the surjective monoid homomorphism $f: R / \sim_{1} \longrightarrow R / \sim_{2}$ given by $f\left([x]_{\sim_{1}}\right)=[x]_{\sim_{2}}$ induces a surjective function $F^{\prime}: \bar{\Gamma}_{\sim_{1}}(R) \longrightarrow \bar{\Gamma}_{\sim_{2}}(R)$ (resp., $F^{\prime \prime}: A G_{\sim_{1}}(R) \longrightarrow A G_{\sim_{2}}(R)$ ) given by $F^{\prime}\left([x]_{\sim_{1}}\right)=[x]_{\sim_{2}}$ (resp., $\left.F^{\prime \prime}\left([x]_{\sim_{1}}\right)=[x]_{\sim_{2}}\right)$ and an injective graph homomorphism $G^{\prime}: \bar{\Gamma}_{\sim_{2}}(R) \longrightarrow \bar{\Gamma}_{\sim_{1}}(R)$ (resp., $G^{\prime \prime}: A G_{\sim_{2}}(R) \longrightarrow A G_{\sim_{1}}(R)$ ) such that $F^{\prime} G^{\prime}=$ $1_{\bar{\Gamma}_{\sim_{2}}(R)}$ (resp., $\left.F^{\prime \prime} G^{\prime \prime}=1_{A G_{\sim_{2}}(R)}\right)$. Moreover, for adjacent vertices $[x]_{\sim_{1}}$ and $[y]_{\sim_{1}}$ in $\bar{\Gamma}_{\sim_{1}}(R)$ (resp., $A G_{\sim_{1}}(R)$ ), either $F^{\prime}\left([x]_{\sim_{1}}\right)=F^{\prime}\left([y]_{\sim_{1}}\right)$ (resp., $F^{\prime \prime}\left([x]_{\sim_{1}}\right)=$ $F^{\prime \prime}\left([y]_{\sim_{1}}\right)$ ) or $F^{\prime}\left([x]_{\sim_{1}}\right)$ and $F^{\prime}\left([y]_{\sim_{1}}\right)$ (resp., $F^{\prime \prime}\left([x]_{\sim_{1}}\right)$ and $F^{\prime \prime}\left([y]_{\sim_{1}}\right)$ ) are adjacent in $\bar{\Gamma}_{\sim_{2}}(R)$ (resp., $A G_{\sim_{2}}(R)$ ); and $\bar{\Gamma}_{\sim_{2}}(R)$ (resp., $A G_{\sim_{2}}(R)$ ) is isomorphic to an induced subgraph of $\bar{\Gamma}_{\sim_{1}}(R)$ (resp., $A G_{\sim_{1}}(R)$ ).

By [12, Theorems 2.8 and 3.1], for a fixed semigroup ideal $I$ of $R$, there is a largest and a smallest $\sim$-zero-divisor graph for $\sim \in \mathcal{C}_{I}(R)$. The largest is $\Gamma_{I}(R)$, and the smallest is $\Gamma_{\sim}(R)$, where $x \sim y \Leftrightarrow(I: x)=(I: y)$. Moreover, $\Gamma_{\sim}(R)=\Gamma_{E}(R / I)$ when $I$ is a proper ideal of $R$ (see Corollary 5.5). In particular, for $I=\{0\}, \Gamma(R)$ is the largest and $\Gamma_{E}(R)$ is the smallest zero-divisor graph. In [9, p. 1450070-4], it was observed that $\Gamma_{E}(R)$ is isomorphic to a subgraph of $\Gamma(R)$, and in [31, Corollary $2.7]$ that $\Gamma_{I}(R)$ contains $|I|$ disjoint subgraphs isomorphic to $\Gamma(R / I)$.

By Theorem 5.2, for $\sim \in \mathcal{C}_{I}(R)$, there are also a largest and a smallest $\bar{\Gamma}_{\sim}(R)$ and $A G_{\sim}(R)$. The largest are $\bar{\Gamma}_{I}(R)$ and $A G_{I}(R)$, and the smallest are $\bar{\Gamma}_{\sim}(R)$ and $A G_{\sim}(R)$, where $x \sim y \Leftrightarrow(I: x)=(I: y)$. Moreover, $\bar{\Gamma}_{\sim}(R)=\bar{\Gamma}_{E}(R / I)$ and $A G_{\sim}(R)=A G_{E}(R / I)$ (see Corollary 5.5). In particular, for $I=\{0\}$, the smallest are the "compressed" graphs $\bar{\Gamma}_{E}(R)$ and $A G_{E}(R)$, and the largest are $\bar{\Gamma}(R)$ and $A G(R)$.

However, for $\sim_{1}, \sim_{2} \in \mathcal{C}_{I}(R)$ with $\sim_{1} \leq \sim_{2}$, the function $F: \Gamma_{\sim_{1}}(R) \longrightarrow \Gamma_{\sim_{2}}(R)$ given by $F\left([x]_{\sim_{1}}\right)=[x]_{\sim_{2}}$ need not be a graph homomorphism since distinct adjacent vertices in $\Gamma_{\sim_{1}}(R)$ may collapse to the same vertex in $\Gamma_{\sim_{2}}(R)$. For example, let $R=\mathbb{Z}_{9}, \sim_{1}==_{R}$, and define $\sim_{2}$ by $x \sim_{2} y \Leftrightarrow \operatorname{ann}_{R}(x)=\operatorname{ann}_{R}(y)$. Then $\sim_{1}$, $\sim_{2} \in \mathcal{C}_{\{0\}}(R)$ with $\sim_{1} \leq \sim_{2}, \Gamma_{\sim_{1}}(R)=\Gamma(R)=K^{2}$, and $\Gamma_{\sim_{2}}(R)=\Gamma_{E}(R)=K^{1}$ (this corrects [12, p. 124]); so $F: \Gamma(R) \longrightarrow \Gamma_{E}(R)$ is not a graph homomorphism. The next theorem gives a sufficient condition for $F, F^{\prime}$, and $F^{\prime \prime}$ to be graph homomorphisms. Note that $\bar{\Gamma}_{\sim}(R)=\Gamma_{\sim}(R)$ when $I=[0]_{\sim}$ is a radical semigroup ideal of $R$.

Theorem 5.3. Let $R$ be a commutative ring with $1 \neq 0, I$ a radical semigroup ideal of $R$, and $\sim_{1}, \sim_{2} \in \mathcal{C}_{I}(R)$ with $\sim_{1} \leq \sim_{2}$. Then $F: \Gamma_{\sim_{1}}(R) \longrightarrow \Gamma_{\sim_{2}}(R)$ (resp., 
$\left.F^{\prime \prime}: A G_{\sim_{1}}(R) \longrightarrow A G_{\sim_{2}}(R)\right)$ given by $F\left([x]_{\sim_{1}}\right)=[x]_{\sim_{2}}$ (resp., $\left.F^{\prime \prime}\left([x]_{\sim_{1}}\right)=[x]_{\sim_{2}}\right)$ is a surjective graph homomorphism.

Proof. The $\Gamma_{\sim}(R)$ case is [12, Theorem 3.2]; so we do the $A_{\sim}(R)$ case. Suppose that distinct vertices $[x]_{\sim_{1}}$ and $[y]_{\sim_{1}}$ are adjacent in $A G_{\sim_{1}}(R)$. Then $(I: x y) \neq(I:$ $x) \cup(I: y)$ for $I=[0]_{\sim_{1}}=[0]_{\sim_{2}}$. Hence there is a $z \in R$ with $z x y \in I$ and $z x, z y \notin I$. If $[x]_{\sim_{2}}=F^{\prime \prime}\left([x]_{\sim_{1}}\right)=F^{\prime \prime}\left([y]_{\sim_{1}}\right)=[y]_{\sim_{2}}$, then $x \sim_{2} y$; so $z x^{2} \sim_{2} z x y \sim_{2} 0$. Thus $z x^{2} \in[0]_{\sim_{2}}=I$, and hence $z x \in I$ since $I$ is a radical semigroup ideal of $R$, a contradiction. Thus $[x]_{\sim_{2}}$ and $[y]_{\sim_{2}}$ are distinct adjacent vertices in $A G_{\sim_{2}}(R)$, and hence $F^{\prime \prime}$ is a surjective graph homomorphism.

In some cases, $\mathrm{a} \sim \in \mathcal{C}_{I}(R)$ induces a $\sim^{\prime} \in \mathcal{C}_{\{0\}}(R / I)$ such that $[x]_{\sim} \mapsto[x+I]_{\sim^{\prime}}$ gives a graph isomorphism $\Gamma_{\sim}(R) \longrightarrow \Gamma_{\sim^{\prime}}(R / I)$ ([12, Theorem 5.1]). Thus a $\sim$-zero-divisor graph may come from different base rings. We next formalize this "change of rings" result to $\bar{\Gamma}_{\sim}(R)$ and $A G_{\sim}(R)$. Note that the " $\sim_{1} \leq \sim$ " hypothesis is needed in Theorem 5.4 (see [12, Remark 5.3]). Again, $F, F^{\prime}$, and $F^{\prime \prime}$ take the same values on $Z(R / \sim)^{*}$.

Theorem 5.4. Let $R$ be a commutative ring with $1 \neq 0$, and let $I$ be a proper ideal of $R$. Define $\sim_{1} \in \mathcal{C}_{I}(R)$ by $x \sim_{1} y \Leftrightarrow x-y \in I$. Given $\sim \in \mathcal{C}_{I}(R)$ with $\sim_{1} \leq \sim$, define $\sim^{\prime} \in \mathcal{C}_{\{0\}}(R / I)$ by $x+I \sim^{\prime} y+I \Leftrightarrow x \sim y$. Then $F: \Gamma \sim(R) \longrightarrow$ $\Gamma_{\sim^{\prime}}(R / I)$ (resp., $F^{\prime}: \bar{\Gamma}_{\sim}(R) \longrightarrow \bar{\Gamma}_{\sim^{\prime}}(R / I), F^{\prime \prime}: A G_{\sim}(R) \longrightarrow A G_{\sim^{\prime}}(R / I)$ ) given by $F\left([x]_{\sim}\right)=[x+I]_{\sim^{\prime}}\left(\right.$ resp., $\left.F^{\prime}\left([x]_{\sim}\right)=[x+I]_{\sim^{\prime}}, F^{\prime \prime}\left([x]_{\sim}\right)=[x+I]_{\sim^{\prime}}\right)$ is a graph isomorphism.

Proof. It is easy to verify that $\sim^{\prime}$ is well-defined and $\sim^{\prime} \in \mathcal{C}_{\{0\}}(R / I)$ since $\sim_{1} \leq$ $\sim$. Define $f: R / \sim(R / I) / \sim^{\prime}$ by $f\left([x]_{\sim}\right)=[x+I]_{\sim^{\prime}}$. It is also easy to verify that $f$ is a monoid isomorphism and induces a graph isomorphism $F: \Gamma_{\sim}(R) \longrightarrow$ $\Gamma_{\sim^{\prime}}(R / I)$ (resp., $F^{\prime}: \bar{\Gamma}_{\sim}(R) \longrightarrow \bar{\Gamma}_{\sim^{\prime}}(R / I), F^{\prime \prime}: A G_{\sim}(R) \longrightarrow A G_{\sim^{\prime}}(R / I)$ ) given by $F\left([x]_{\sim}\right)=[x+I]_{\sim^{\prime}}\left(\operatorname{resp} ., F^{\prime}\left([x]_{\sim}\right)=[x+I]_{\sim^{\prime}}, F^{\prime \prime}\left([x]_{\sim}\right)=[x+I]_{\sim^{\prime}}\right)$, i.e., $F=F^{\prime}=F^{\prime \prime}=\left.f\right|_{Z(R / \sim)^{*}}$.

Corollary 5.5. Let $R$ be a commutative ring with $1 \neq 0$, and let $I$ be a proper ideal of $R$. Define $\sim_{1}, \sim_{2} \in \mathcal{C}_{I}(R)$ by $x \sim_{1} y \Leftrightarrow x-y \in I$ and $x \sim_{2} y \Leftrightarrow(I: x)=$ $(I: y)$. Then $\Gamma_{\sim_{1}}(R)=\Gamma(R / I), \bar{\Gamma}_{\sim_{1}}(R)=\bar{\Gamma}(R / I), A G_{\sim_{1}}(R)=A G(R / I)$, and $\Gamma_{\sim_{2}}(R)=\Gamma_{E}(R / I), \bar{\Gamma}_{\sim_{2}}(R)=\bar{\Gamma}_{E}(R / I), A G_{\sim_{2}}(R)=A G_{E}(R / I)$.

Most of the results in [12, Sections 5 and 6$]$ for $\Gamma_{\sim}(R)$ extend in the natural way to $\bar{\Gamma}_{\sim}(R)$ and $A G_{\sim}(R)$ since all three graphs have the same vertex set. We leave the routine details to the interested reader. 


\section{References}

[1] M. Afkhami, N. Hoseini and K. Khashyarmanesh, The annihilator ideal graph of a commutative ring, Note. Mat., 36(1) (2016), 1-10.

[2] M. Afkhami, K. Khashyarmanesh and Z. Rajabi, Some results on the annihilator graph of a commutative ring, Czechoslovak Math. J., 67(1) (2017), 151-169.

[3] M. Afkhami, K. Khashyarmanesh and S. M. Sakhdari, The annihilator graph of a commutative semigroup, J. Algebra Appl., 14(2) (2015), 1550015 (14 pp).

[4] D. D. Anderson and M. Naseer, Beck's coloring of a commutative ring, J. Algebra, 159(2) (1993), 500-514.

[5] D. F. Anderson, On the diameter and girth of a zero-divisor graph, II, Houston J. Math., 34(2) (2008), 361-371.

[6] D. F. Anderson, M. C. Axtell and J. A. Stickles, Jr., Zero-divisor graphs in commutative rings, in Commutative Algebra, Noetherian and Non-Noetherian Perspectives (M. Fontana, S.-E. Kabbaj, B. Olberding, I. Swanson, Eds.), Springer-Verlag, New York, (2011), 23-45.

[7] D. F. Anderson and A. Badawi, The zero-divisor graph of a commutative semigroup: a survey, Groups, modules, and model theory - surveys and recent developments, Springer, Cham, (2017), 23-39.

[8] D. F. Anderson and J. D. LaGrange, Commutative Boolean monoids, reduced rings, and the compressed zero-divisor graph, J. Pure Appl. Algebra, 216(7) (2012), 1626-1636.

[9] D. F. Anderson and J. D. LaGrange, Abian's poset and the ordered monoid of annihilator classes in a reduced commutative ring, J. Algebra Appl., 13(8) (2014), 1450070 (18 pp).

[10] D. F. Anderson and J. D. LaGrange, Some remarks on the compressed zerodivisor graph, J. Algebra, 447 (2016), 297-321.

[11] D. F. Anderson, R. Levy and J. Shapiro, Zero-divisor graphs, von Neumann regular rings, and Boolean algebras, J. Pure Appl. Algebra, 180(3) (2003), 221-241.

[12] D. F. Anderson and E. F. Lewis, A general theory of zero-divisor graphs over a commutative ring, Int. Electron. J. Algebra, 20 (2016), 111-135.

[13] D. F. Anderson and P. S. Livingston, The zero-divisor graph of a commutative ring, J. Algebra, 217(2) (1999), 434-447.

[14] D. F. Anderson and S. B. Mulay, On the diameter and girth of a zero-divisor graph, J. Pure Appl. Algebra, 210(2) (2007), 543-550. 
[15] S. E. Atani, A. Y. Darani and E. R. Puczylowski, On the diameter and girth of ideal based zero-divisor graphs, Publ. Math. Debrecen, 78(3-4) (2011), 607-612.

[16] A. Badawi, On 2-absorbing ideals of commutative rings, Bull. Austral. Math. Soc., 75(3) (2007), 417-429.

[17] A. Badawi, On the annihilator graph of a commutative ring, Comm. Algebra, 42(1) (2014), 108-121.

[18] I. Beck, Coloring of commutative rings, J. Algebra, 116(1) (1988), 208-226.

[19] D. Bennis, J. Mikram and F. Taraza, On the extended zero divisor graph of commutative rings, Turkish J. Math., 40(2) (2016), 376-388.

[20] B. Bollobás, Graph Theory, An Introductory Course, Graduate Texts in Mathematics, 63, Springer-Verlag, New York-Berlin, 1979.

[21] J. Coykendall, S. Sather-Wagstaff, L. Sheppardson and S. Spiroff, On zero divisor graphs, in Progress in Commutative Algebra II: Closures, Finiteness and Factorization (C. Francisco et al., Eds.), Walter de Gruyter, Berlin, (2012), 241-299.

[22] F. R. DeMeyer, T. McKenzie and K. Schneider, The zero-divisor graph of a commutative semigroup, Semigroup Forum, 65(2) (2002), 206-214.

[23] F. DeMeyer and K. Schneider, Automorphisms and zero-divisor graphs of commutative rings, Internat. J. Commutative Rings, 1(3) (2002), 93-106.

[24] S. Dutta and C. Lanong, On annihilator graphs of a finite commutative ring, Trans. Comb., 6(1) (2017), 1-11.

[25] R. Gilmer, Commutative Semigroup Rings, Chicago Lectures in Mathematics, The University of Chicago Press, Chicago, 1984.

[26] J. A. Huckaba, Commutative Rings with Zero Divisors, Monographs and Textbooks in Pure and Applied Mathematics, 117, Marcel Dekker, Inc., New York, 1988.

[27] I. Kaplansky, Commutative Rings, Revised edition, The University of Chicago Press, Chicago, 1974.

[28] G. McClurken, Generalizations and Variations of the Zero-Divisor Graph, Doctoral dissertation, The University of Tennessee, August 2017.

[29] S. B. Mulay, Cycles and symmetries of zero-divisors, Comm. Algebra, 30(7) (2002), 3533-3558.

[30] Sh. Payrovi and S. Babaei, The compressed annihilator graph of a commutative ring, Indian J. Pure Appl. Math., 49(1) (2018), 177-186.

[31] S. P. Redmond, An ideal-based zero-divisor graph of a commutative ring, Comm. Algebra, 31(9) (2003), 4425-4443. 
[32] J. G. Smith, Jr., When ideal-based zero-divisor graphs are complemented or uniquely complemented, Int. Electron. J. Algebra, 21 (2017), 198-204.

[33] S. Spiroff and C. Wickham, A zero divisor graph determined by equivalence classes of zero divisors, Comm. Algebra, 39(7) (2011), 2338-2348.

\section{David F. Anderson}

Department of Mathematics

The University of Tennessee

Knoxville, TN 37996, U. S. A.

e-mail: danders5@utk.edu

Grace McClurkin (Corresponding Author)

Department of Mathematics

Saginaw Valley State University

University Center, MI 48710, U. S. A.

e-mail: gmcclurk@svsu.edu 